\title{
Loss-of-function CARD8 mutation causes NLRP3 inflammasome activation and Crohn's disease
}

\author{
Liming Mao, ${ }^{1}$ Atsushi Kitani, ${ }^{1}$ Morgan Similuk, ${ }^{2}$ Andrew J. Oler, ${ }^{3}$ Lindsey Albenberg, ${ }^{4}$ Judith Kelsen, ${ }^{4}$ Atiye Aktay, ${ }^{5}$ \\ Martha Quezado, ${ }^{6}$ Michael Yao, ${ }^{1,7}$ Kim Montgomery-Recht, ${ }^{8}$ Ivan J. Fuss, ${ }^{1}$ and Warren Strober ${ }^{1}$ \\ 'Mucosal Immunity Section, Laboratory of Clinical Immunology and Microbiology (LCIM), National Institute of Allergy and Infectious Diseases (NIAID), NIH, Bethesda, Maryland, USA. ${ }^{2}$ Clinical Genomics \\ Program, NIAID, NIH, Bethesda, Maryland, USA. ${ }^{3}$ Bioinformatics and Computational Biosciences Branch, Office of Cyber Infrastructure and Computational Biology, NIAID, NIH, Bethesda, Maryland, USA. \\ ${ }^{4}$ Division of Pediatric Gastroenterology, Children's Hospital of Philadelphia, Philadelphia, Pennsylvania, USA. 5Division of Pediatric Gastroenterology, University Hospitals Cleveland Medical Center, Cleveland, \\ Ohio, USA. 'aboratory of Pathology, National Cancer Institute (NCI), NIH, Bethesda, Maryland, USA. 'Division of Gastroenterology, Washington DC VA Medical Center, Washington DC, USA. ${ }^{8}$ Clinical Research \\ Directorate/Clinical Monitoring Research Program, Leidos Biomedical Research Inc., NCI Campus at Frederick, Frederick, Maryland, USA.
}

\begin{abstract}
In these studies, we evaluated the contribution of the NLRP3 inflammasome to Crohn's disease (CD) in a kindred containing individuals having a missense mutation in CARD8, a protein known to inhibit this inflammasome. Whole exome sequencing and PCR studies identified the affected individuals as having a V44I mutation in a single allele of the T60 isoform of CARD8. The serum levels of IL-1 $\beta$ in the affected individuals were increased compared with those in healthy controls, and their peripheral monocytes produced increased amounts of IL-1 $\beta$ when stimulated by NLRP3 activators. Immunoblot studies probing the basis of these findings showed that mutated T60 CARD8 failed to downregulate the NLRP3 inflammasome because it did not bind to NLRP3 and inhibit its oligomerization. In addition, these studies showed that mutated T60 CARD8 exerted a dominant-negative effect by its capacity to bind to and form oligomers with unmutated T60 or T48 CARD8 that impeded their binding to NLRP3. Finally, inflammasome activation studies revealed that intact but not mutated CARD8 prevented NLRP3 deubiquitination and serine dephosphorylation. CD due to a CARD8 mutation was not effectively treated by anti-TNF- $\alpha$, but did respond to IL- $1 \beta$ inhibitors. Thus, patients with anti-TNF- $\alpha$-resistant CD may respond to this treatment option.
\end{abstract}

\section{Introduction}

The influence of genetic factors in the pathogenesis of inflammatory bowel disease (IBD) was made dramatically evident by the identification of numerous IBD-associated risk polymorphisms, i.e., SNPs in various genetic regions that occur in greater frequency in patients with IBD than in the general population (1). These risk polymorphisms, in keeping with and supporting the hyperresponsive concept of IBD pathogenesis, quite frequently involve genes affecting inflammatory responses (1). In recent years, several publications have appeared highlighting the possible pathogenetic importance of one such inflammation-associated gene, that encoding NLRP3 $(2,3)$. NLRP3 is a pattern-recognition receptor that initiates the activation of the NLRP3 inflammasome, a protein complex that regulates the generation of mature forms of IL-1 $\beta$ and IL-18 and the induction of inflammatory cell death known as pyroptosis (4). However, while factors that induce either excess or diminished NLRP3 inflammasome activity have indeed been shown to affect gastrointestinal (GI) inflammation, it has not been shown that the NLRP3 inflammasome can act on its own to induce such inflammation. It appears that in most cases, NLRP3 inflammasome activity is one of an array of proinflam-

Conflict of interest: The authors have declared that no conflict of interest exists Submitted: November 20, 2017; Accepted: January 30, 2018. Reference information: J Clin Invest. 2018;128(5):1793-1806. https://doi.org/10.1172/JCI98642. matory factors causing GI inflammation in Crohn's disease (CD) (see further discussion below).

One characteristic of the NLRP3 inflammasome that might limit its effect on CD inflammation is that this source of proinflammatory cytokines is tightly controlled by several naturally occurring negative regulators. CARD8, one such negative regulator expressed in humans and not in rodents, is the focus of the present study.

As shown by Ito et al., CARD8 binds to NLRP3 during inflammasome activation and, in doing so, prevents its binding to its downstream adaptor ASC and thereby the assembly of the NLRP3 inflammasome complex (5). In addition, these investigators showed that NLRP3 with mutations associated with cryopyrinassociated periodic syndromes (CAPS) increased inflammasome activity and lost the ability to bind to CARD8. Additional evidence of CARD8 inhibition of NLRP3 has come from studies by Cheung et al., who reported that a frameshift mutation in CARD8, resulting in loss of binding to NLRP3, caused a form of CAPS marked by aphthous stomatitis, pharyngitis, and cervical adenitis (periodic fever, aphthous stomatitis, pharyngitis, adenitis [PFAPA] syndrome) (6). These studies introduce the possibility that a loss-offunction mutation in CARD8 could be associated with increased NLRP3 inflammasome activity.

Previous studies of a possible CARD8 abnormality affecting the occurrence of CD have been concerned with a CARD8 polymorphism at rs2043211 that causes a stop codon in exon 5 of CARD8 (C10X) and therefore prevents transcription of the T48 
isoform of CARD8 $(7,8)$. However, while this SNP was associated with Crohn's disease in several studies involving a limited number of patients it was not so associated in studies involving a large number of patients (9). These more negative studies may be due to the fact that most patients bearing this polymorphism continue to express a slightly smaller but nevertheless fully functional isoform (T47) as a result of transcription of a CARD8 isoform that initiates downstream of the C10X site (10).

In this study, we report a kindred containing several individuals carrying a CARD8 mutation at V44I upstream of the T48 start site and therefore expressed in the T60 CARD8 isoform (see diagram in Figure 1A). All 3 individuals carrying this mutation developed a CD-like intestinal inflammation associated with extensive evidence of NLRP3 inflammasome activation. The latter consisted of high levels of circulating IL- $1 \beta$ and the presence of peripheral monocytes exhibiting increased NLRP3 activation. The mutant CARD8 lacked the ability to interact with NLRP3 and inhibit NLRP3 oligomerization. More importantly, intestinal inflammation in the proband persisted despite a full range of biologic and immunosuppressive therapy until the proband was treated with anakinra or an IL-1 $\beta$-neutralizing antibody. These data thus provide the first evidence, to our knowledge, that a mutation affecting a component of the NLRP3 inflammasome, leading to its excessive activation, can be a cause of CD in the absence of other abnormalities.

\section{Results}

Clinical courses of patients in a kindred with a CARD8 mutation and $C D$-like intestinal inflammation. A kindred containing 3 members bearing a CARD8 mutation is depicted in Figure 1A. In all 3 of these members, the proband, his mother, and his maternal aunt, the mutation occurred in association with CD-like intestinal inflammation (Figure 1, B-I); see detailed description of the case histories of the proband, his mother, and his maternal aunt in Supplemental Methods (supplemental material available online with this article; https://doi.org/10.1172/JCI98642DS1). The proband's father did not carry the CARD8 mutation and was free of GI disease. Of note, the proband did not improve upon treatment with steroids plus anti-TNF- $\alpha$ and, while initial histopathologic examination showed changes found in graft-versus-host disease (GvHD), follow-up examination after some clinical improvement showed changes indicative of CD. He was therefore administered anakinra (IL-1 receptor antagonist), a treatment that led to decreased diarrhea and other GI symptoms and was accompanied by a sharp decrease in the fecal calprotectin level (see Figure 1J). This suggested the presence of excessive NLRP3 inflammasome activity and led to the studies detailed below.

DNA-sequencing data of individuals in the kindred. Whole exome sequencing identified a number of variants in the patients who composed the above kindred (Supplemental Figure 1). Among these, the variant chr19:48741719 C>T (hg19) in CARD8 stood out because of its role in the inflammasome $(5,6)$. This variant has an allele frequency of $0.0015 \%$ in the genome Aggregation Database (gnomAD; http://gnomad.broadinstitute.org/) (2 heterozygous individuals), is predicted to be possibly damaging/ deleterious by PolyPhen (http://genetics.bwh.harvard.edu/pph/ data/) and SIFT (http://sift.jcvi.org/), and has a CADD-PHRED score (http://cadd.gs.washington.edu/info) of 11.3, which is above the mutation significance cutoff score (11) of 3.3 for CARD8. This variant overlaps multiple transcripts, so in the context of the gene, the variant could be in the intron, in the $5^{\prime}$ UTR, or be a V44I missense mutation (Supplemental Figure 2). However, we detected no difference in CARD8 expression at the mRNA and protein levels in cells from the proband compared with cells from a healthy control (Supplemental Figure 3, A and B), so the only relevant annotation is a missense mutation at $\mathrm{V} 44 \mathrm{I}$.

In related studies in which DNA fragments generated by PCR using primers surrounding the CARD8 mutation site were sequenced, the presence of a single allele V44I mutation in DNA from both the proband and his mother (Supplemental Figure 4) was again observed; in addition, these studies also revealed the same CARD8 mutation in a maternal aunt who also has CD (Supplemental Figure 4). Finally, while these sequencing studies were performed on DNA obtained from peripheral cells, quantitative PCR (qPCR) and Western blot studies of small intestine and colonic tissues showed that CARD8 bearing the V44I mutation was expressed at substantial levels in proband GI tissue (Supplemental Figure 5).

Additional analysis of the above sequencing data revealed another variant of potential interest, a missense $\mathrm{R} 329 \mathrm{H}$ variant in the $M E F V$ gene having a population allele frequency of $0.2 \%$. The $M E F V$ gene encodes pyrin, a protein that forms the pyrin inflammasome with ASC and caspase- 1 that releases IL- $1 \beta$ when stimulated by its activator TcdB. However, we did not find excessive pyrin inflammasome activity in the proband (Supplemental Figure 6). The $M E F V$ variant occurred only in the proband and his mother, not in the proband's aunt with well-defined CD, who also bears the CARD8 V44I mutation (Supplemental Figure 4). This suggests that the $M E F V$ variant does not contribute to the development of $\mathrm{CD}$ in this kindred.

Yet another fact to emerge from the above sequencing data was that DNA from neither the proband nor his parents bore a SNP at rs2043211 in exon 6 (GenBank) (https://www.ncbi.nlm.nih. gov/nuccore/NG_029574.2 ?from $=5324 \&$ to $=53184 \&$ report $=$ genbank) of CARD8, a polymorphism that has been shown to occur at an increased frequency in patients with $\operatorname{CD}$ in some studies $(7,8)$.

CARD8 is known to be expressed as one of several isoforms with differing transcription start sites leading to differing molecular weights. This includes a T48 isoform with a start site in exon 6 and a larger T60 isoform with an upstream start site in exon 4 (see Figure 1K). The latter is the isoform that contains the Card8 mutation at V44I and therefore is the isoform that is potentially responsible for the functional effects of the mutation.

Finally, it should be noted that a gene equivalent to CARD8 is not found in mice. Thus, studies of the effects of mutations in the various isoforms of this gene are limited to in vitro studies of human cells that cannot be further elucidated by in vivo studies of similar CARD8 mutants in mice.

The CARD8 V44I mutation is associated with enhanced IL-1 production and increased NLRP3 inflammasome activation. Recent studies have provided evidence that the CARD8 T48 isoform has an inhibitory effect on NLRP3 inflammasome activation (5). To determine whether the CARD8 T60 isoform, i.e., the isoform that bears a V44 site capable of bearing a V44I mutation, has a similar effect on NLRP3 inflammasome regulation, we transfected plasmids express- 
A

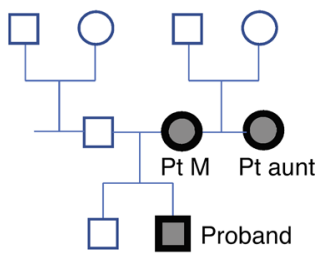

O Active Crohn's Disease

Mutation carrier

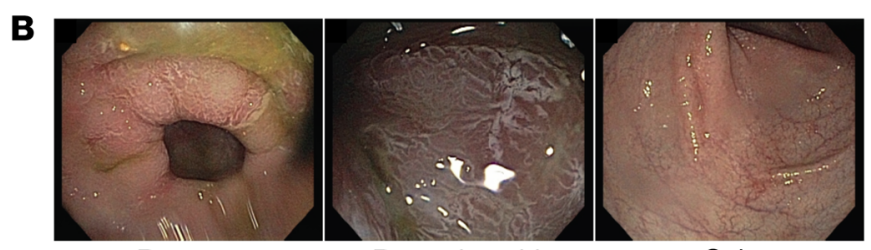

Rectum
Rectosigmoid
Colon
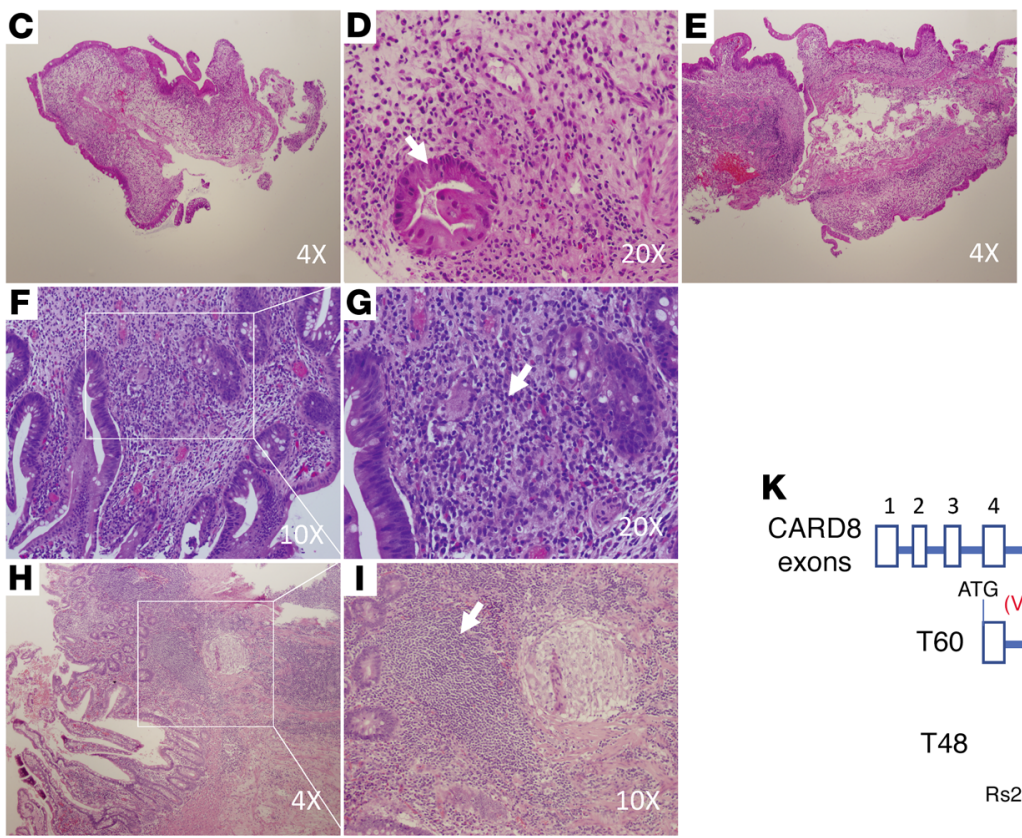

I
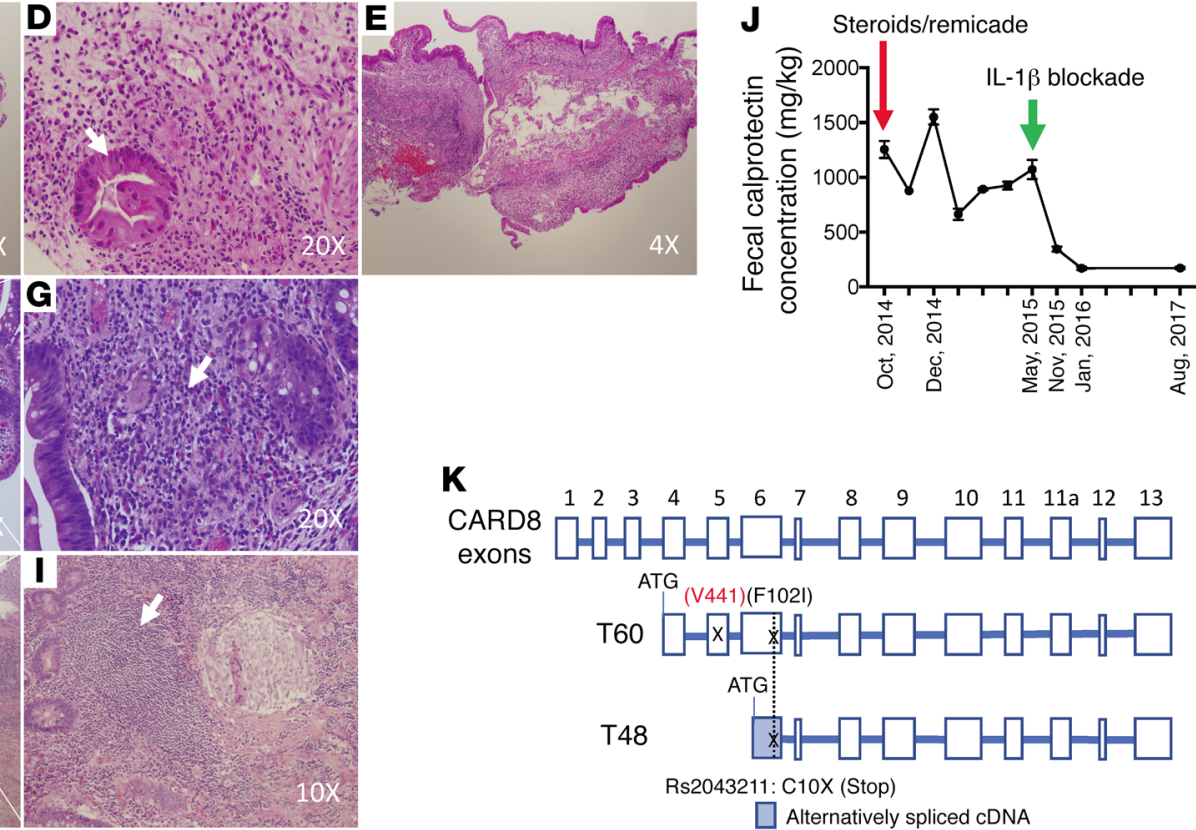

Figure 1. A kindred with family members bearing a CARD8 mutation and CD-like intestinal inflammation. (A) A kindred containing 3 members bearing a CARD8 mutation. In all 3 members of the kindred, the proband (Pt), his mother (Pt M), and his maternal aunt (Pt Aunt), the mutation occurred in association with a CD-like intestinal inflammation. (B) Macroscopic examination of the colon exhibited scattered areas of superficial erythema and ulceration having a lenticular pattern especially evident in the rectosigmoid region. $n=3$ /group. (C-I) Biopsies from the terminal ileum and colon. (C) Index patient colon. Colitis with epithelial erosive changes and inflammation, significant crypt and goblet cell loss with regenerative changes. Features consistent with GvHD. (D) Index patient colon. Colitis with rare residual gland showing goblet cell loss, repair changes, and rare apoptotic bodies (arrow). (E) Index patient terminal ileum. Ileitis with focal erosion, villi loss, lymphocytic infiltrates, severe crypt drop-out, and repair changes. (F) Index patient terminal ileum. Chronic active ileitis with regenerative changes and poorly formed granulomas including giant cells. (G) Index patient terminal ileum. Poorly formed granuloma (arrow) with giant cells. Adjacent glands with repair/regenerative changes. (H) Aunt terminal ileum. Transmural lymphocyte infiltration with well-formed granuloma present. (I) Aunt terminal ileum. Well-formed granuloma (arrow). $n=3$ /group. Original magnification, $\times 4$ (C, E, H); $\times 10$ (F, I); $\times 20$ (D, G). Parts G and I show higher magnification of boxed areas in $\mathbf{F}$ and $\mathbf{H}$, respectively. (J) Anakinra therapy resulted in rapid clinical improvement marked by decreased fecal calprotectin levels. Data are representative of 3 independent experiments. (K) Whole exome sequencing revealed a CARD8 V44I mutation in 1 allele of chromosome 19 (see sequencing data in the text). The mutation site of V44I was present on the CARD8 T60 isoform, but not the "canonical" T48 isoform.

ing human NLRP3 together with plasmids expressing CARD8 T60, CARD T48, or empty vector into HEK293 cells and, 24 hours later, transfected the cells with plasmids expressing ASC, caspase-1, and pro-IL-1 $\beta$ to allow assembly of an NLRP3 inflammasome. Then, after another 24 hours, the cells were stimulated with nigericin and the culture medium of incubated cells was collected for assessment of IL-1 $\beta$ secretion. We found that cells transfected with plasmids expressing either CARD8 T60 or T48 isoforms produced significantly reduced levels of IL-1 $\beta$ compared with cells transfected with empty vector (Figure 2A). These data thus provided evidence that the intact (unmutated) CARD8 T60 isoform also plays an inhibitory role during NLRP3 inflammasome activation. In addition, they suggested that a loss-of-function CARD8 mutation in CARD8 T60 could be affecting this inhibitory role.
In initial studies to examine this latter possibility, we determined concentrations of IL-1 $\beta$ and IL- 6 in a random sample of proband serum by ELISA and found that concentrations of both of these cytokines were clearly increased compared with levels in sex-matched control sera (Figure 2B). In related studies, we determined whether proband peripheral blood mononuclear cells (PBMCs) or monocytes produce increased amounts of IL-1 $\beta$, as might be expected if the NLRP3 inflammasome was dysregulated by the mutation. Accordingly, we cultured PBMCs and purified proband monocytes for 24 or 48 hours in the absence of stimulation and then determined the IL-1 concentration in the culture supernatants. We found IL-1 $\beta$ in the supernatants from both proband cell cultures was significantly increased compared with that in supernatants of control cell cultures (Figure 2C). 
A

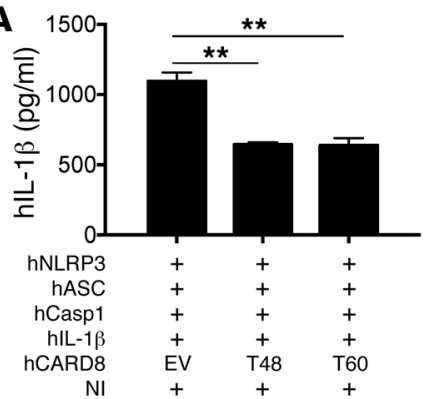

C

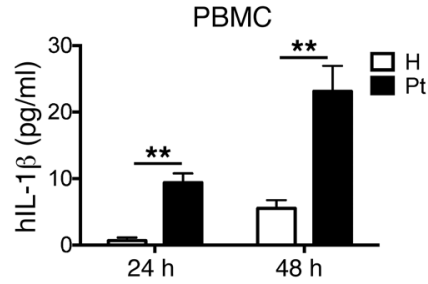

D

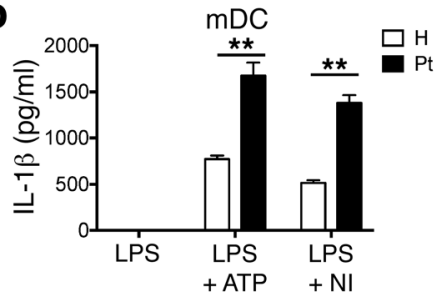

B
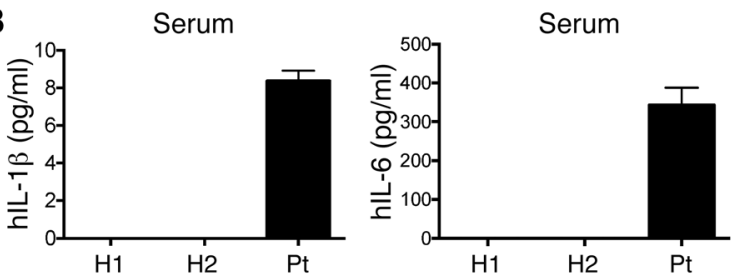
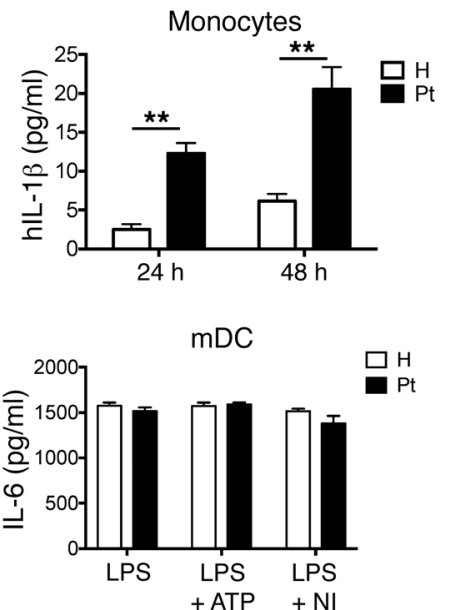

$\mathbf{E}$

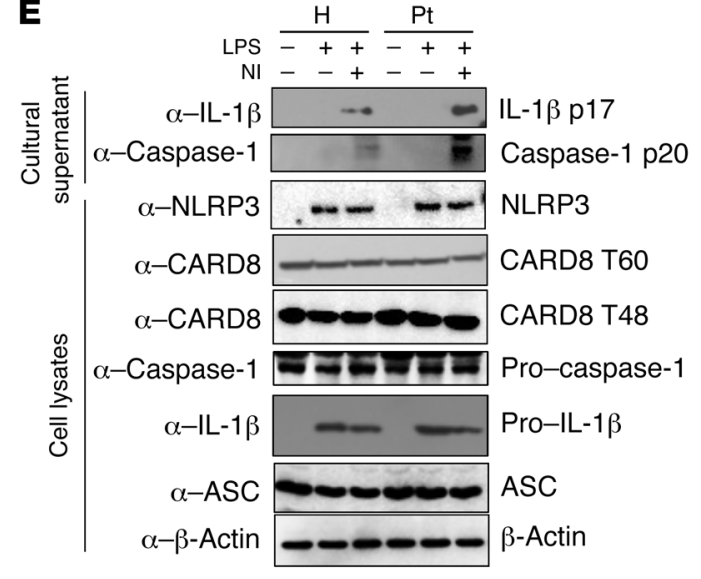

Figure 2. The CARD8 V44I mutation is associated with enhanced IL-1ß production and increased NLRP3 inflammasome activation. (A) HEK293 cells were transfected with plasmids carrying human NLRP3 together with CARD8 T48 or T60 or empty vector (EV) and, 24 hours later, transfected with plasmids carrying ASC, caspase-1. and pro-IL-1 $\beta$ (to allow assembly of the NLRP3 inflammasome). Twenty-four hours later, cells were stimulated with nigericin (NI) for 30 minutes, after which culture supernatants were collected for IL-1 $\beta$ ELISA assay. Data are shown as mean \pm SEM. ** $P<0.01$, 1-way ANOVA with Dunnett's post hoc test. (B) Serum samples from the proband (with a CARD8 V44I mutation) and age- and sex-matched healthy controls (H1 and H2) were collected and subjected to IL-1 and IL-6 ELISA assays. (C) Proband and control PBMCs and monocytes were cultured for 24 or 48 hours without stimulation, after which supernatants were subjected to IL-1 $\beta$ ELISA assay. Data are shown as mean \pm SEM. $n=3$. ${ }^{* *} P<0.01,2$-tailed Student's $t$ test. (D) Proband and control mDCs were primed with LPS $(100 \mathrm{ng} / \mathrm{ml})$ for 6 hours and stimulated with ATP $(5 \mathrm{mM})$ or nigericin (1.2 $\mu \mathrm{M})$ for 30 minutes, after which culture supernatants were collected and assayed for IL-1 $\beta$ and IL- 6 by ELISA. Data are shown as mean \pm SEM. $n=3$. ${ }^{* *} P<0.01,2$-tailed Student's $t$ test. (E) Western blot detection of mature IL-1 $\beta$ and mature caspase-1. Data for ELISA are representative of 3 independent experiments. Data for Western blot are representative of 2 independent experiments. See complete unedited blots in the supplemental material.

With these data in hand, we next conducted studies to formally determine whether the CARD8 V44I mutation in the CARD8 T60 isoform affects NLRP3 inflammasome activation. We therefore examined IL-1 $\beta$ production by proband and control peripheral monocyte-derived dendritic cells (mDCs) stimulated by LPS alone or LPS in the presence of NLRP3 activators, ATP, or nigericin. We found that cells from the proband stimulated under these conditions produced significantly more IL-1 $\beta$ (Figure 2D) and IL-18 (Supplemental Figure 7) than cells from control individuals, but produced similar amounts of IL-6 (Figure 2D). In addition, in Western blot studies, we found that the cells from the proband produced greatly increased amounts of mature IL-1 $\beta$ and mature caspase- 1 than control cells (Figure 2E). Moreover, the proband's mother and maternal aunt carrying the CARD8 V44I mutation also displayed overactivity of the NLRP3 inflammasome (Supplemental Figures 8 and 9). During the study, peripheral blood specimens from different heathy individuals were used as controls, and in all cases, IL-1 $\beta$ production was lower than that observed in patients with the CARD8 mutation. These data thus suggested that the V44I mutation negated the inhibitory role of CARD8 on NLRP3 inflammasome activation.

The CARD8 mutation does not affect pyrin or NLRC4 inflammasome activation, but has a marginal effect on AIM inflammasome activation. Since intact CARD8 may regulate one or more inflammasomes other than the NLRP3 inflammasome, it was possible that a loss-of-function mutation in CARD8 causes increased IL-1 $1 \beta$ production via dysregulation of several inflammasomes. To investigate this possibility, we primed purified $\mathrm{mDCs}$ from proband and control cells with LPS and then stimulated these mDCs with ATP, TcdB, transfected poly(dA:dT), or flagellin to activate the NLRP3, pyrin, AIM2, or NLRC4 inflammasomes, respectively. Our results showed that, as expected, the mDCs from the proband produced more IL-1 $\beta$ than those from control cells after NLRP3 activation with LPS/ATP stimulation (Figure $3 \mathrm{~A}$ ). In addition, proband $\mathrm{mDCs}$ stimulated with the AIM inflammasome activator polyAdT also produced a greater amount of IL-1 $\beta$ than control cells, although in this case, the increase, while repeatable, was relatively small compared with that obtained by the activation of the NLRP3 inflammasome (Figure 3B). In con- 
A

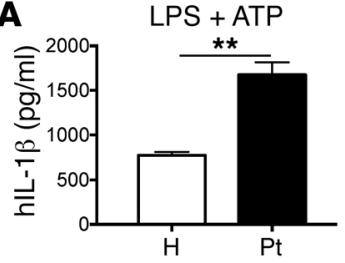

E

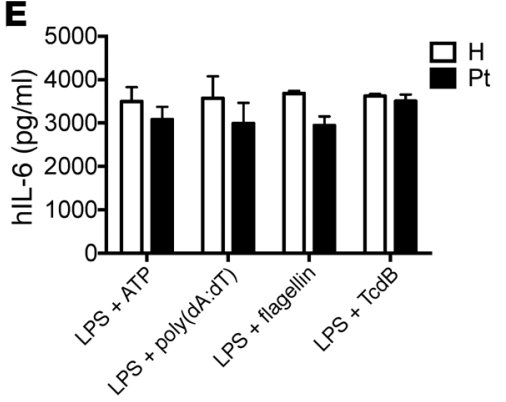

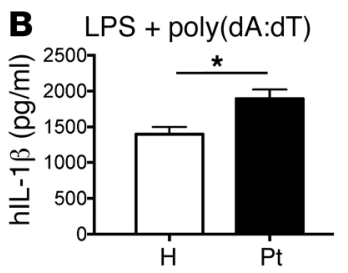
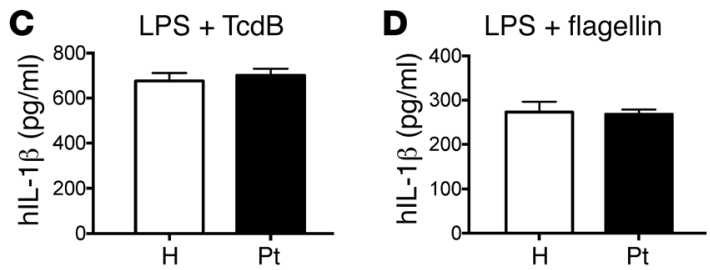

$\mathbf{F}$

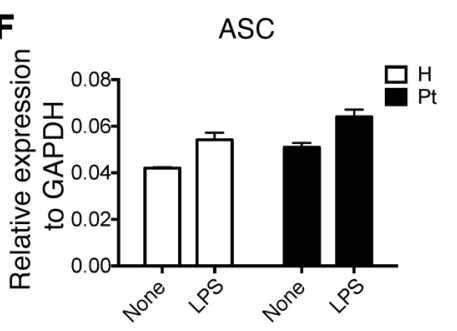

${ }^{\circ}$
G Caspase-1

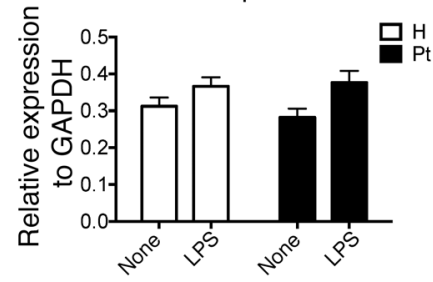

H
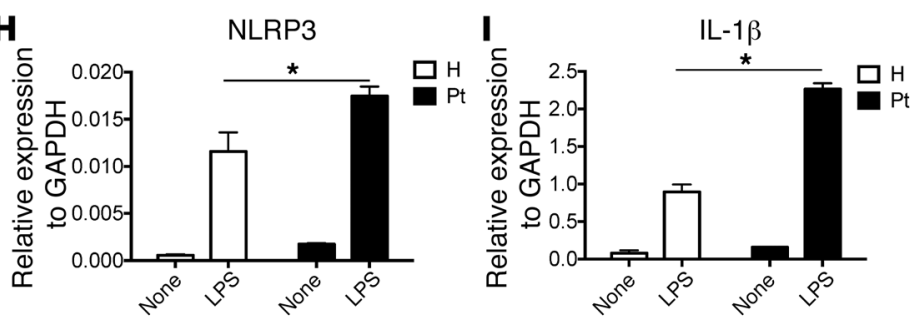

Figure 3. The CARD8 mutation affects NLRP3 and AIM2 inflammasome, but not pyrin and NLRC4 inflammasome, activation. mDCs from the proband and a healthy control were primed with LPS (100 ng/ml, 6 hours) and then stimulated with ATP (5 mM, A), poly(dA:dT) (1 $\mu \mathrm{g} / \mathrm{ml}, 2 \mathrm{hours}, \mathbf{B})$, TcdB ( $1 \mu \mathrm{g} / \mathrm{ml}$, 2 hours, $\mathbf{C})$, or flagellin $(1 \mu \mathrm{g} / \mathrm{ml}, 2$ hours, D) to activate the NLRP3, AIM2, pyrin, or NLRC4 inflammasomes, respectively. Culture supernatants were collected and subjected to IL-1 $\beta$ (A-D) and IL-6 (E) assay by ELISA. Primary mDCs from proband and healthy control were treated with or without LPS (100 ng/mI) for 6 hours, after which cells were harvested and subjected to RNA extraction. Quantitative reverse-transcriptase PCR (qRT-PCR) of the extracted RNA was performed to determine the expression of ASC (F), caspase-1 (G), NLRP3 (H), and pro-IL-1 $\beta(\mathbf{I})$. Data are shown as mean \pm SEM. $n=3$. ${ }^{*} P<0.01 ;{ }^{*} P<0.05$, 2 -tailed Student's $t$ test. All data are representative of 3 independent experiments.

trast, the production of IL-1 $\beta$ was comparable between patient and control cells after TcdB or flagellin stimulation (Figure 3, C and D). These differential responses were specific to IL-1 $\beta$, as each of the stimuli resulted in equal IL-6 responses (Figure 3E). Together, these data demonstrated that the CARD8 V44I mutation mainly enhances NLRP3 inflammasome activation, and perhaps the AIM2 inflammasome, but does not affect the pyrin or NLRC4 inflammasome. This is most likely attributable to the fact that CARD8 does not regulate these inflammasome even in the intact state.

CARD8 with a V44I mutation exhibits reduced binding to NLRP3 and affects NLRP3 inflammasome assembly. To investigate the mechanism by which the CARD8 V44I mutation exhibits decreased inhibition of the NLRP3 inflammasome, we first asked whether this mutation affects the transcription of the genes related to the NLRP3 inflammasome and thus alters their expression. To attempt to answer this question, we assessed expression levels of various NLRP3 components in MDCs from the proband as compared with those in $\mathrm{mDCs}$ from a healthy control individual. We found that proband cells expressed comparable levels of ASC and caspase- 1 (Figure 3, F and G), but significantly increased levels of NLRP3 and IL-1 $\beta$ (Figure 3, H and I). The increase in NLRP3 mRNA associated with the CARD8 V44I mutation is presently unexplained, but may be due to the fact that the mutation negates known inhibitory effects of CARD8 on NF- $\mathrm{KB}$ activation and thereby causes an increase in NLRP3 transcription (12).
Next, we determined whether the mutation in CARD8 affects its ability to bind to NLRP3. To this end, HEK293 cells transfected with plasmids expressing intact CARD8 T60 or CARD8 T60 bearing the V44I mutation along with a plasmid expressing NLRP3 were cultured for 48 hours, after which cell lysates were prepared and subjected to immunoprecipitation with anti-CARD8 antibody followed by immunoblotting with anti-NLRP3 antibody. The results showed that the interaction between mutant CARD8 T60 and NLRP3 was remarkably reduced compared with the interaction between intact CARD8 T60 and NLRP3 (Figure 4A). In parallel studies, we determined whether a similar effect can be observed under physiological conditions. In these studies, we stimulated mDCs from the proband and a healthy control with LPS or LPS plus nigericin and then, after an appropriate incubation period, prepared cell lysates and subjected the latter to immunoblot studies as discussed above. We found that intact CARD8 bound to NLRP3 when stimulated with LPS, but not after LPS and nigericin treatment (Figure 4B). In contrast, the mutant CARD8 did not bind to NLRP3 in any circumstance (Figure 4B). These data indicate that CARD 8 bearing the V44I mutation has a reduced capacity to bind to NLRP3 under physiologic conditions.

Despite the fact that the proband does not express the CARD8 SNP at rs2043211 that has been associated with CD in some studies $(7,8)$, it was of interest to determine whether this SNP also causes an abnormality of the capacity of CARD8 to bind to 
A

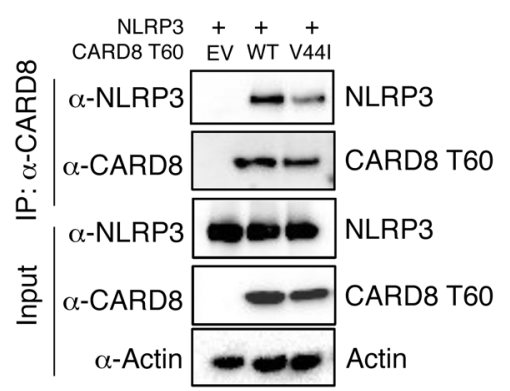

D

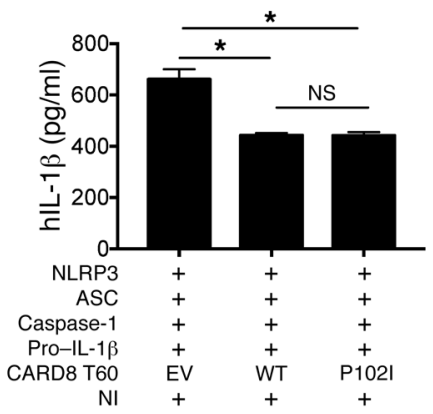

B

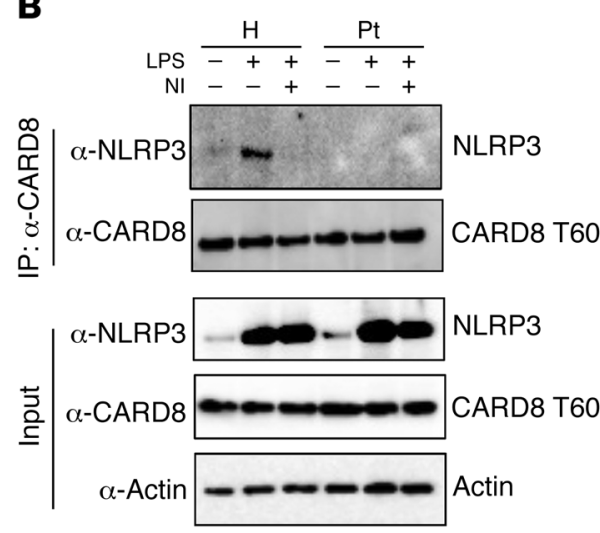

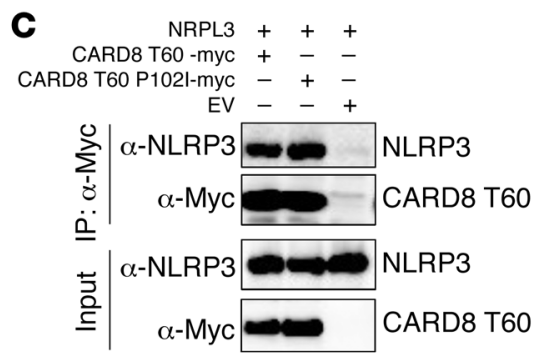

E

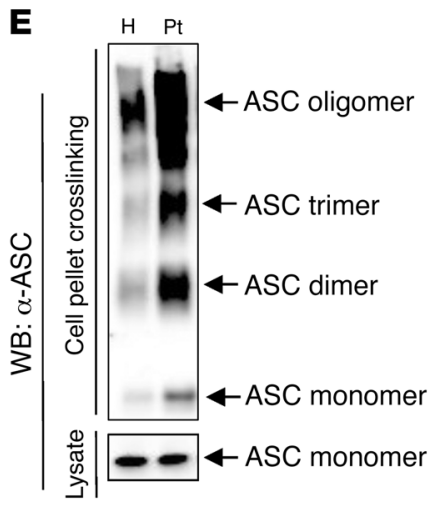

$\mathbf{F}$

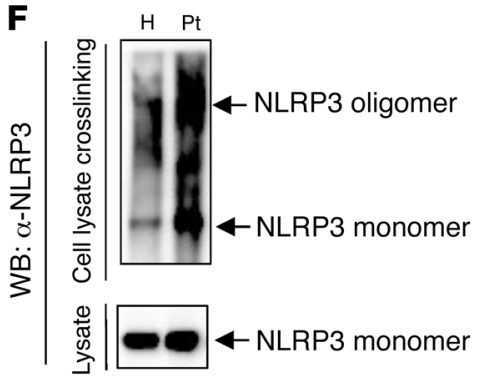

Figure 4. CARD8 with a V44I mutation exhibits reduced binding to NLRP3 and affects NLRP3 inflammasome assembly. (A) Plasmids expressing intact CARD8, CARD8 V44I mutation, or empty vector were transfected into HEK293 cells along with a plasmid expressing NLRP3. At 48 hours after transfection, cells were harvested and cell lysates were subjected to immunoprecipitation using anti-CARD8 antibody, followed with immunoblotting. (B) mDCs from proband and healthy control were stimulated with LPS ( $100 \mathrm{ng} / \mathrm{ml}, 6$ hours) or LPS plus nigericin $(1.2 \mu \mathrm{M}, 30$ minutes). Cells were lysed, and lysates were subjected to immunoprecipitation and immunoblotting. (C) HEK293 cells were transfected with a plasmid expressing NLRP3 along with Myc-tagged intact CARD8 T60, CARD8 T60 P102I, or empty vector. Cells were lysed at 48 hours, and lysates were subjected to immunoprecipitation using anti-Myc antibody, followed by immunoblotting. (D) HEK293 cells were transfected with NLRP3 plasmid along with Myc-tagged intact CARD8 T60, CARD8 T60 P102I, or empty vector. Twenty-four hours later, cells were transfected with plasmids expressing ASC, caspase-1, and pro-IL-1 $\beta$ to allow assembly of the NLRP3 inflammasome. Another 24 hours later, cells were stimulated with nigericin $(1.2 \mu \mathrm{M}, 30$ minutes). The cultural supernatants were examined for IL-1 $\beta$ concentration by ELISA. Data are shown as mean \pm SEM. ${ }^{*} P<0.05$, 1-way ANOVA with Dunnett's post hoc test. (E) mDCs from proband and a healthy control were stimulated with LPS (100 ng/ml, 6 hour) plus nigericin ( $1.2 \mu \mathrm{M}, 30$ minutes). Cells were prepared for Western blot as in Methods. (F) Cell lysates shown in $\mathbf{E}$ were prepared for Western blot as in Methods. Data for ELISA are representative of 3 independent experiments. Data for Western blot are representative of 2 independent experiments. See complete unedited blots in the supplemental material.

NLRP3, as does the V44I mutation. Whereas this SNP introduces a stop codon $(\mathrm{C} 10 \mathrm{X})$ in the T48 isoform of CARD8 (and therefore the likely formation of a downstream transcriptional start site for this isoform), it causes a P102I mutation in the T60 isoform of CARD8 due to the upstream start site governing this isoform. Accordingly, to determine the effect of this SNP on CARD8 binding to NLRP3, we transfected plasmids expressing intact or P102Imutated T60 CARD8 together with a plasmid expressing NLRP3 into HEK293 cells and then performed immunoblotting studies to examine CARD8/NLRP3 interactions. We found that the interaction between NLRP3 and CARD8 T60 P102I was comparable to that with intact CARD8 T60 (Figure 4C). In an additional study in which we determined the capacity of CARD8 T60 P102I or intact CARD8 T60 to inhibit an assembled NLRP3 inflammasome in HEK293 cells as described above, we observed that P102I and intact CARD8 had comparable abilities to inhibit the secretion of IL-1 $\beta$ by the NLRP3 inflammasome (Figure 4D). Thus, even in individuals carrying a homozygous SNP at rs2043211, the CARD8 isoform T60 still regulates NLRP3 inflammasome activity.

Finally, we investigated whether the lack of CARD8 binding to NLRP3 associated with the mutation affects NLRP3 inflammasome assembly. For this purpose, we determined whether the mutation affects ASC polymerization and perinuclear speck formation, an obligate downstream effect of its binding to NLRP3, and inflammasome assembly and activation. We found that mDCs from the peripheral blood of the proband with a CARD8 V44I mutation exhibited increased ASC oligomerization upon NLRP3 inflammasome activation with LPS and nigericin as compared with cells from a control individual (Figure 4E). In addition, oligomerization of NLRP3 was also increased in cells with the CARD8 V44I mutation (Figure $4 \mathrm{~F}$ ). These results indicate that reduced CARD8 function due to a V44I mutation is accompanied by increased NLRP3 assembly.

CARD8 bearing the V44I mutation has a dominant-negative effect. As noted above, the CARD8 V44I mutation is found on 
A
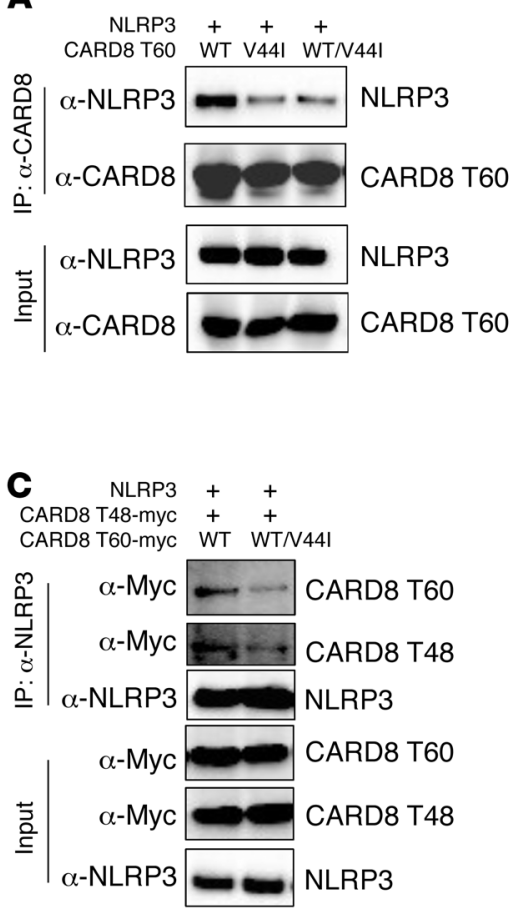

Figure 5. Mutant CARD8 exerts a dominant-negative effect on the NLRP3 inflammasome. (A) HEK293 cells were transfected with plasmids expressing intact and mutant CARD8 T60 alone or together (half of the amount per plasmids, to mimic the heterozygous status of the proband). After 48 hours of incubation, cell lysates were obtained and subjected to immunoprecipitation and immunoblotting to determine CARD8 binding to NLRP3. (B) HEK293 cells were transfected with NLRP3 and Flag-tagged CARD8 T48 plasmids along with intact or mutant CARD8 T60 plasmids. After 48 hours, cell lysates were obtained and subjected to immunoprecipitation (using antiCARD8 antibody) and immunoblotting. (C) HEK293 cells were transfected with NLRP3 and CARD8 T48 plasmids along with intact or mutant CARD8 T60 plasmids. After 48 hours, cell lysates were obtained and subjected to immunoprecipitation (using anti-NLRP3 antibody) and immunoblotting. (D) HEK293 cells were transfected with NLRP3 and intact CARD8 T48 or T60 alone or together with mutant CARD8 T60 plasmids. Twenty-four hours later, T cells were transfected with ASC, caspase-1, and pro-IL-1 $\beta$ plasmids to allow the assembly of NLRP3 inflammasome. Twenty-four hours later, cells were stimulated with nigericin $(1.2 \mu \mathrm{M})$ for 30 minutes. Cultural supernatants were collected for IL-1 $\beta$ ELISA assay. Data are shown as mean $\pm \mathrm{SEM}$. ${ }^{*} P<0.05,1$-way ANOVA with Tukey's post hoc test. Data for ELISA are representative of 3 independent experiments. Data for Western blot are representative of 2 independent experiments. See complete unedited blots in the supplemental material.

only 1 allele of the proband and is therefore capable of producing intact CARD8 (albeit in reduced amounts). It therefore seemed likely that the mutated CARD8 was exerting a dominant-negative effect and thereby inhibiting the function of the intact CARD8. To examine this possibility, we transfected plasmids expressing mutated and intact $\mathrm{T} 60$ isoforms of CARD8 alone or together into HEK293 cells and then performed immunoblot studies to determine CARD8 binding to NLRP3, as described above. We found that cotransfection of mutated CARD8 with intact CARD8 greatly decreased CARD8 and NLRP3 binding (Figure 5A).

Next, to determine whether the interaction between CARD8 T48 and NLRP3 was affected by the CARD8 T60 V44I mutation, we transfected HEK293 cells with a plasmid expressing the T48 isoform of CARD8 together with a plasmid expressing the intact or mutated T60 isoform of CARD8 and then performed immunoblot studies as described above. We found that the presence of mutated CARD8 T60, but not intact CARD8 T60, reduced T48 cells transfected with intact CARD8 T60/mutated CARD8 T60 mixtures exhibited strong oligomer formation (Figure 6, C and D). Similarly, intact CARD8 T48/T60 exhibited relatively poor oligomer formation, whereas intact CARD8 T48/mutated CARD8 T60 exhibited robust oligomer formation (Figure 6E). These data thus suggest that mutated CARD8 exerts a dominant-negative effect by binding to intact CARD8 isoforms, thereby disrupting the latter's ability to bind to NLRP3 (see model in Figure 6F).

The CARD8 V44I mutation results in reduced NLRP3 serine phosphorylation as well as reduced K63 and K48 polyubiquitination. Recent studies have provided evidence that serine phosphorylation of NLRP3 mediated by phosphokinase A (PKA) inhibits NLRP3 inflammasome activation and that such phosphorylation is disrupted in CAPS patients with increased NLRP3 inflammasome activity (13). In addition, it has been reported that NLRP3 polyubiquitination also inhibits NLRP3 inflammasome activation $(14,15)$. It is therefore possible that the inhibitory effect of intact CARD8 and 


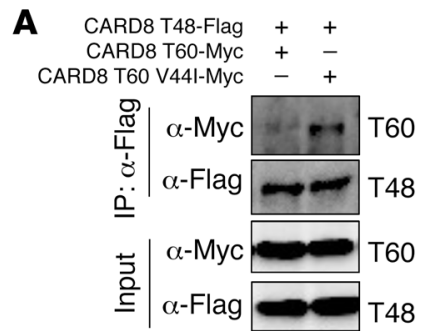

D

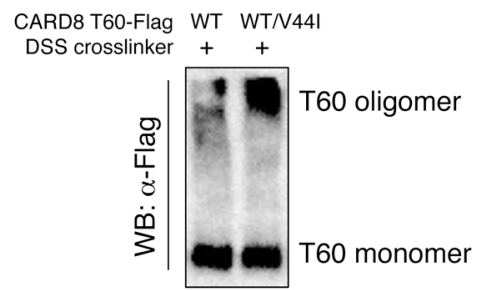

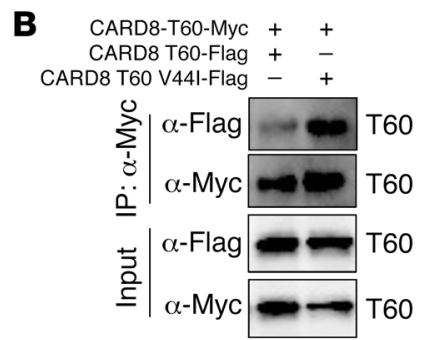

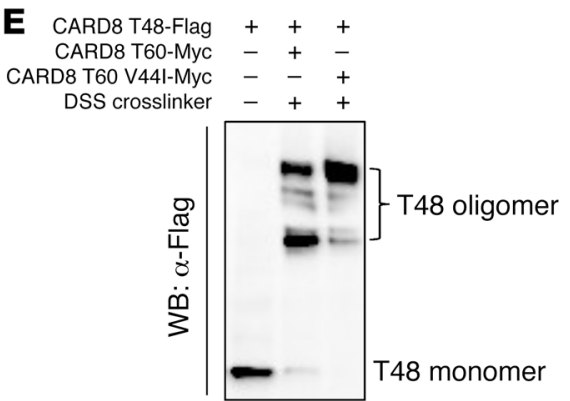

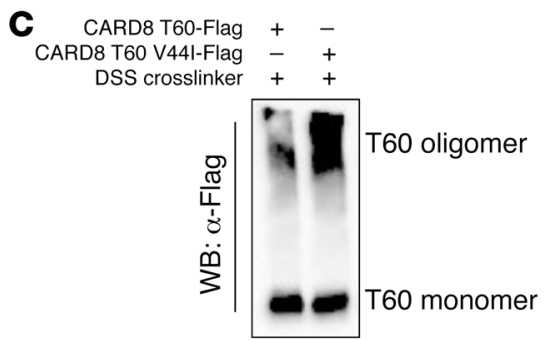

F WT CARD8

Mutant CARD8

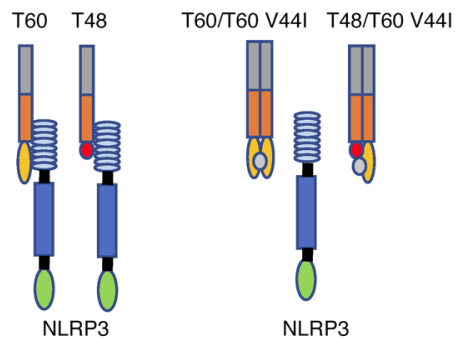

Figure 6. Mutant CARD8 T60 disrupts interaction between T48 and NLRP3. (A) HEK293 cells were transfected with Flag-tagged CARD8 T48 and with Myc-tagged intact or mutant CARD8 T60. After 48 hours, cell lysates were obtained and subjected to immunoprecipitation (with anti-Flag antibody), followed by immunoblotting. (B) HEK293 cells were transfected with Myc-tagged CARD8 T60 along with Flag-tagged intact or mutant CARD8 T60 plasmids. After 48 hours, cell lysates were obtained and subjected to immunoprecipitation (using anti-Myc antibody), followed by immunoblotting. (C) HEK293 cells were transfected with Flag-tagged intact or mutant CARD8 T60 plasmids. After 48 hours, cell lysates were obtained and treated with DSS crosslinker and then with LDS loading buffer for Western blot. (D) HEK293 cells were transfected with Flag-tagged intact CARD8 T60 plasmid alone or together with a mutant CARD8 T60 plasmid. After 48 hours, cell lysates were obtained and treated with DSS crosslinker and then with LDS loading buffer for Western blot. (E) HEK293 cells were transfected with a Flag-tagged CARD8 T48 along with intact or mutant CARD8 T60 plasmids: after 48 hours, cell lysates were obtained and treated with DSS crosslinker and then with LDS loading buffer for Western blot. (F) Diagram showing that mutant CARD8 T60 exhibits increased binding with intact T60 and T48 and that this binding is thought to block interaction between intact CARD8 T60 or T48 with NLRP3. All data are representative of 2 independent experiments. See complete unedited blots in the supplemental material.

its loss in the presence of a V44I CARD8 mutation is due to effects on NLRP3 serine phosphorylation and NLRP3 polyubiquitination.

To examine this possibility, we transfected plasmids expressing human NLRP3, ASC, caspase-1, and either intact or mutated CARD8 into HEK293 cells to determine the effect of CARD8 or mutant CARD8 on serine phosphorylation of NLRP3 during its activation in a reassembled NLRP3 inflammasome. We found that serine phosphorylation of immunoprecipitated NLRP3 (detected with a phosphoserine-specific antibody) in cells with expression of intact CARD8 was increased compared with that in cells without transfection of CARD8 (Figure 7A). In addition, the phosphorylation of NLRP3 in cells transfected with mutated CARD8 was reduced compared with that in cells transfected with intact CARD8 (Figure 7A). However, after treatment with nigericin, NLRP3 phosphorylation in both samples was reduced (Figure 7A). These data suggest that before activation, NLRP3 was in a serinephosphorylated state, allowing the NLRP3 inflammasome to maintain an inactive status; however, when stimulated by its activators, the phosphorylation was reduced and thus allowed activation. In contrast, the mutated CARD8, presumably due to its lack of binding, resulted in reduced phosphorylation of NLRP3 even prior to activation and therefore led to enhanced activation.

In parallel studies to verify these findings under physiologic conditions, we pretreated mDCs from the proband and a control individual with LPS and then activated the NLRP3 inflammasome with nigericin. Finally, we subjected cell lysates from these cells to immunoprecipitation with anti-NLRP3 antibody and then immunoblotting with anti-phosphoserine antibody as described above. In this case, we found that cells with the CARD8 mutation exhibited remarkably less NLRP3 serine phosphorylation than cells with intact CARD8 (Figure 7B). Taken together, these studies show that CARD8 regulates NLRP3 inflammasome activation at the level of serine phosphorylation and that the CARD8 mutation affects this regulation.

We next asked whether intact or mutated CARD8 affects polyubiquitination of NLRP3. To this end, we transfected constructs carrying human NLRP3, ASC, caspase-1, or intact or mutated CARD8 T60 together with constructs expressing K63 or K48 ubiquitin chain into HEK293 cells. The cells were then incubated for 48 hours, after which cell lysates were subjected to immunoprecipitation with anti-NLRP3 antibody and Western blot to examine the polyubiquitination of NLRP3. We found that the K63- and K48-linked polyubiquitination of NLRP3 in cells transfected with CARD8 T60 V44I mutant were both reduced compared with those in cells transfected with intact CARD8 T60 (Figure 7C). These studies therefore imply that CARD8 also regulates NLRP3 inflammasome at the level of polyubiquitination and that the CARD8 mutation affects this regulation.

Taken together, these results suggest that the overactivation of NLRP3 inflammasome in the CD patients bearing mutated CARD8 is caused by both reduced NLRP3 phosphorylation and K63- and K48-linked polyubiquitination of NLRP3. 
A

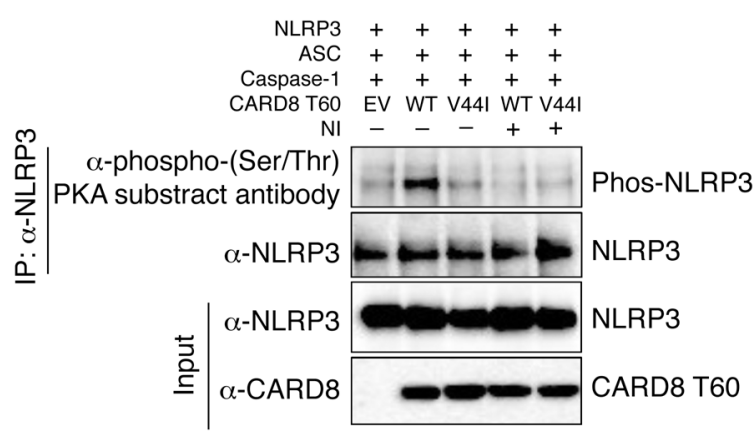

B

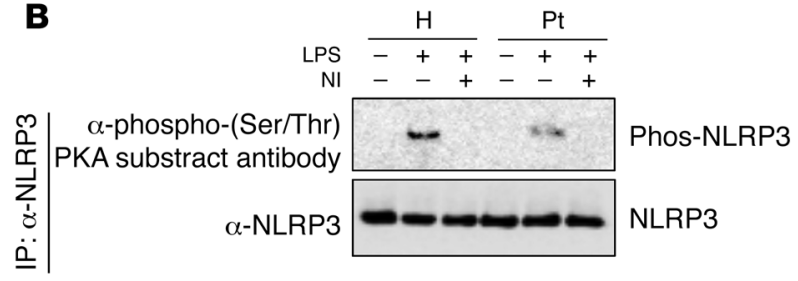

C



Figure 7. The CARD8 V44I mutation results in reduced NLRP3 serine phosphorylation as well as reduced K63 and K48 polyubiquitination. (A) HEK293 cells were transfected with human NLRP3, ASC, caspase-1, and either intact or mutant CARD8 T60 plasmids. After 48 hours, cells were stimulated with or without nigericin (1.2 $\mu \mathrm{M}, 30$ minutes). Cell lysates were obtained and subjected to immunoprecipitation (with anti-NLRP3 antibody) and immunoblotted with anti-phosphoserine antibody. (B) mDCs from the proband and a healthy control were pretreated with LPS (100 ng/ml, 6 hours) and then activated with nigericin ( $1.2 \mu \mathrm{M}, 30$ minutes). Cell lysates from these cells were subjected to immunoprecipitation and immunoblotting as described above. (C) HEK293 cells were transfected with NLRP3, ASC, and caspase-1 plasmids as well as intact or mutant CARD8 T60 plasmids together with constructs expressing K63 or K48 ubiquitin chains. Forty-eight hours later, cell lysates were obtained and subjected to immunoprecipitation (with anti-NLRP3 antibody) and Western blot to examine the polyubiquitination of NLRP3. The top row image was spliced from images obtained from the same gel, but with different exposure times. All data are representative of 2 independent experiments. See complete unedited blots in the supplemental material.

$I L-1 \beta$ antibody administration is an effective form of treatment of $C D$ in a patient bearing a CARD8 V44I mutation. As noted above, $\mathrm{CD}$ in the proband bearing the CARD8 mutation was greatly ameliorated by treatment targeting IL-1 $\beta$ (anakinra and canakinumab). We therefore performed studies to determine the effect of this treatment on NLRP3 inflammasome function. We found that the antibody treatment reduced serum IL- $1 \beta$ as well as IL- 6 and TNF- $\alpha$ to levels observed in control individuals (Figure 8, A-C). However, stimulation of circulating monocytes under NLRP3-activating conditions revealed that the antibody had no effect on inflammasome activation and that the V44I mutation was still capable of inducing increased activation. The effect of antibody treatment was therefore directed at the output of the NLRP3 inflammasome activation rather than at its activation.

The above data showed that increased IL- $1 \beta$ secretion played a pathogenic role in the proband and that the concentration of IL-1 $\beta$ in the circulation was correlated with disease activity. This prompted us to ask how circulating IL-1 $\beta$ levels in the patient with the CARD8 mutation compared with those in conventional CD patients without a CARD8 mutation. To this end, we collected serum samples from 4 patients with moderately active $C D$ and compared the circulating IL-1 $\beta$, IL- 6 , and TNF- $\alpha$ levels in these patients with those in the patient with the CARD8 mutation and healthy individuals. The results showed that the concentration of all 3 cytokines was significantly higher than that in healthy controls (Figure 8, D-F), but lower than in the patient with the CARD8 mutation. This suggests that, whereas conventional CD patients have increased NLRP3 inflammasome activity, the latter is not as elevated as in the patient with the CARD8 mutation. This is probably one of the reasons why GI disease in the patient with the mutation was not responsive to treatment that did not address NLRP3 hyperactivity.

\section{Discussion}

CARD8 (also known as TUCAN or CARDINAL) is a component of the NLRP3 inflammasome that has been shown to negatively regulate its activation and capacity to process IL-1 $\beta$ (5). Here, we report that a V44I mutation in the T60 isoform of CARD8 interferes with its regulatory function and causes a form of $C D$ due to enhanced NLRP3 activity and its secretion of IL-1 $\beta$. This is thus the first report, to our knowledge, showing that an abnormality of IL-1 $\beta$ secretion can be a primary cause of GI inflammation.

The CARD8 mutation was present in 3 members of a kindred, all of whom had CD. Extensive studies of the proband revealed that he exhibited high levels of circulating IL-1 $\beta$ associated with increased NLRP3 inflammasome activation. Mechanistically, this was caused by reduced capacity of the mutated CARD8 to interact with and inhibit NLRP3 oligomerization. Strong evidence that the mutation and its effect on NLRP3 activity was the cause of $\mathrm{CD}$ came from the fact that the proband did not respond clinical- 

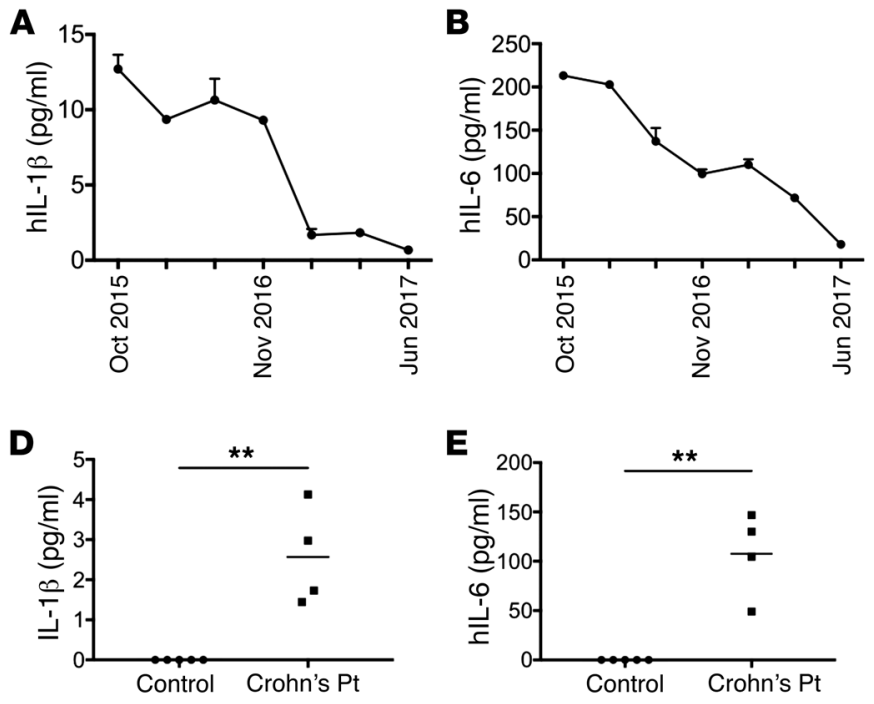

ly to anti-TNF- $\alpha$ and/or immunosuppressive treatment, but did respond to anakinra or anti-IL-1 $\beta$ antibody administration. Studies of the proband's mother and maternal aunt also revealed high circulating levels of IL-1 $\beta$ and increased NLRP3 inflammasome activity, but in these cases, possible clinical responses to anti-IL-1 $\beta$ administration have not been tested. These data on the relation of a CARD8 mutation to causation of CD is parallel to a recent finding that a frameshift mutation in CARD8, also leading to reduced binding of CARD8 with NLRP3, is a cause of periodic fever with aphthous stomatitis, pharyngitis, and cervical adenitis (6).

The CARD 8 gene is present on chromosome 19 and is capable of generating several isoforms of CARD8 that result from alternative N-terminal transcription start sites. The "canonical" T48 isoform has a start site in exon 6 and was shown initially to negatively regulate NF- $\mathrm{BB}$ and promote apoptosis $(12,16)$. Somewhat later it was shown to interact with NLRP3 via its FIIND domain and to negatively regulate the NLRP3 inflammasome (5). Our studies show that a somewhat larger isoform of CARD8, the T60 isoform having a start site in exon 4, also binds to NLRP3 and inhibits its activity. As discussed further below, this is an important finding because the T60 isoform of CARD8, but not the T48 isoform of CARD8, bears the V44I mutation and, therefore, only this isoform can mediate the effect of the mutation on CARD8 regulatory function.

The V44I mutation is a single allele mutation and is therefore present with a normal allele capable of producing intact CARD8 isoforms with normal regulatory function. The T60 isoform of CARD8 bearing the mutation was therefore likely to be acting in a dominant-negative fashion. In studies to explain this dominant-negative effect, we showed first that the cotransfection of mutated CARD8 T60 diminishes the binding of unmutated CARD8 T48 or T60 to bind to NLRP3. In addition, cotransfection of mutated CARD8 T60 with unmutated CARD8 T48 or T60 reduces the ability of either form of CARD8 to downregulate NLRP3 inflammasome-mediated secretion of IL-1 $\beta$. Interestingly, this is associated with the fact that mutated CARD8 T60 has a much greater ability to bind to either CARD8 T60 or CARD8 T48 and to form oligomers with the unmutated isoforms. This supports the idea that mutated CARD8 T60 exerts a dominant-negative

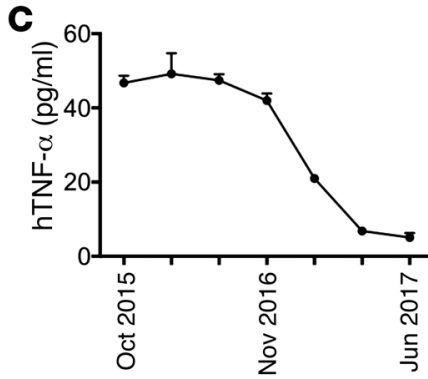

Figure 8. IL-1 $\beta$ antibody treatment reduced peripheral cytokine levels in proband bearing a CARD8 V44I mutation. Seven serum samples from the proband at various time points were collected and subjected to assays of IL- $1 \beta$ (A), IL-6 (B), and TNF- $\alpha$ (C) concentrations by ELISA. Data in $\mathbf{A}-\mathbf{C}$ are shown as mean \pm SEM. Serum samples from patients with active CD without CARD8 V44I mutations and healthy individuals were collected and subjected to assays of the concentration of IL- $1 \beta$ (D), IL-6 (E), and TNF- $\alpha$ (F) by ELISA. CD patients, $n=4$; control, $n=5$. Data in $\mathbf{D}-\mathbf{F}$ are shown as mean \pm SEM. ${ }^{* *} P<0.01,2$-tailed Student's $t$ test. All data are representative of 3 independent experiments. effect by forming oligomers with intact CARD8 isoforms with a diminished ability to bind to NLRP3.

An understanding of how the above changes in CARD8 produced as a result of the mutation lead to the above changes in its binding patterns will require additional structure-based studies. These studies will have to first resolve the fact that both the T60 and T48 isoforms of CARD8 interact with NLRP3 via their N-terminal FIIND domains despite the fact that the T60 FIIND domain is longer than the T48 FIIND domain. Perhaps more importantly, it will have to explain how the mutation of the T60 isoform at V44 causes a change in the FIIND region that produces a CARD8 that is unable to interact with NLRP3 as well as a change that produces a CARD8 more able to interact with unmutated T60 and T48 CARD8 isoforms present in the heterozygous patients that thus block their ability to bind to NLRP3. We speculate that the structural changes affecting interisoform binding involve C-terminal CARD-CARD interactions; thus, the same sequence variant that is unable to bind to NLRP3 via its FIIND domain achieves increased binding to unmutated CARD8 via its CARD domain.

Studies of patients with IBD have established that IL-1 $\beta$ secretion is an integral component of the cytokine milieu driving the colonic inflammation and, in fact, IL- $1 \beta$ levels correlate with disease severity (17-19). However, since IL-1 $\beta$ secretion is one among several proinflammatory factors driving gut inflammation, it has not itself been clearly identified as a primary cause of disease. Studies of murine models of colitis addressing this question, usually of dextran sulfate sodium-induced colitis (DSS-colitis), are inconclusive, since this form of colitis phenocopies human IBD rather poorly, particularly with regard to its dependence on loss of epithelial cell integrity. In any case, in utilizing these models, it has usually been shown that loss-of-function abnormalities of the NLRP3 inflammasome, the main source of IL-1 $\beta$, are associated with more rather than less intestinal inflammation (20-24). One possible explanation of this somewhat paradoxical result is that the NLRP3 inflammasome also generates protective factors, such as antiinflammatory cytokines and IL-18, the latter a cytokine that helps maintain epithelial cell integrity and whose absence therefore leads to increased colitis, especially in the DSS-colitis model 
(21-23). This view is compatible with a recent study in which it was shown that a particular microRNA (miR-223) has inhibitory effects on NLRP3 function and that its absence in mice leads to increased DSS-colitis. Of interest, miR-223 levels are increased in CD patients (and in DSS-colitis), but this increase does not preclude the development of robust inflammation (25). These various studies of animal models in which decreased NLRP3 activity is shown not to lead to decreased intestinal inflammation are complemented by a study in which it was shown that increased NLRP3 activity does not lead to increased inflammation. Thus, in a study by Yao et al., it was shown that mice bearing a mutation in the NLRP3 gene resulting in NLRP3 with increased function similar to that occurring in the autoinflammatory Muckle-Wells syndrome were resistant to DSS-colitis. This was due to IL-1 $\beta$ induction of antibacterial peptides promoting the development of an intestinal microflora containing an organism (Larus marinus) that induces regulatory $\mathrm{T}$ cells in the mucosa capable of suppressing inflammation (26).

The above studies of animal models suggesting that IL-1 $\beta$ is not normally the sole driver of gut inflammation do not rule out the possibility that this cytokine can play an increased proinflammatory role under circumstances in which it is produced in excess and is associated with other proinflammatory conditions. This is shown in studies of mice with abnormalities of IL-10 production or signaling, where IL-10 has been shown to have a profound inhibitory effect on NLRP3 inflammasome activity; therefore, the absence of IL-10 leads to increased IL-1 $\beta$ production (27). In these studies, it has been shown that IL-10-KO and IL-10R-KO mice exhibit increased NLRP3 inflammasome activity and that an NLRP3 inflammasome inhibitor, such as glyburide or caspase-1 inhibitor, ameliorates colitis occurring in such mice $(28,29)$. In addition, in related studies, it has been shown that blockade of IL-10 signaling causes colitis in TLR5-KO mice attributable to IL-1 $\beta$, since it does not occur in TLR5/IL-1 $\beta$ R double-KO mice (30). Finally, and perhaps most importantly, it has been demonstrated that NLRP3 inflammasome hyperactivity and increased IL-1 $\beta$ secretion may mediate colitis in CD patients due to IL-10R deficiency. Therefore, these patients can successfully be treated with the IL-1R blocker anakinra (31). This evidence of a negative relation between IL-10 and the NLRP3 inflammasome may have a direct relevance to the gut inflammation in patients with the CARD8 mutation reported here, as it may be that the presence of a subtle IL-10 deficiency enhances the effect of the mutation on NLRP3 function and thus determines whether the mutation will cause inflammation.

Mutations leading to IL-10 or IL-10R deficiency are not the only genetic defects having possible effects on NLRP3 inflammasome activation. It has been shown, for instance, that several gene polymorphisms associated with increased risk for development of $\mathrm{CD}$, most notably loss-of-function polymorphisms involving NOD2 and ATG16L1, have been associated with changes in NLRP3 activity and could thus be contributors to gut inflammation via their effect on inflammasome function (32). In addition, patients with intestinal inflammation associated with gene abnormalities' leading to mevalonate kinase deficiency and chronic granulomatous disease (CGD) have benefited from treatment with agents that block IL-1 $\beta(33,34)$. Thus, increased production of IL-1 $\beta$ due to a variety of genetic abnormalities may contribute to gut inflammation. Finally, it should be mentioned that active NLRP3 inflammasome activity also results in secretion of IL-18 and, while this cytokine may reduce inflammation by contributing to epithelial integrity as mentioned above, it also may have proinflammatory properties of it own. This is seen in patients with NLRC4 abnormalities that do not respond to IL-1 $\beta$ blockade, but do respond to a combination of agents that block both IL- $\beta$ and IL-18 (35).

The occurrence of a CD phenotype in the proband and his relatives bearing the CARD8 mutation and manifesting increased NLRP3 activity, but not in CAPS patients who bear NLRP3 mutations that also cause increased NLRP3 activity, raises questions about the genetic and/or environmental factors necessary for disease appearance. One possible explanation is that these 2 genetically determined diseases are associated with 1 or more modifying genes that determine the capacity of IL- $1 \beta$ to mediate disease based on the downstream mechanisms of IL-1 $\beta$ activity. Another possible explanation is based on the presence or absence of an environmental factor, such as a microbiome-related development of suppressor cells, that prevents the development of GI disease. This possibility is supported by the recent finding already mentioned above (26), showing that, in mice, a CAPS-like mutation in NLRP3 leading to increased NLRP3 inflammasome activity results in the appearance of a gut organism capable of inducing the development of suppressor T cells. Further work will be necessary to establish these or other explanations of intestinal inflammation in the face of NLRP3 overactivity.

As discussed in the Introduction, a CARD8 polymorphism at rs2043211 has been associated with CD in some studies (7, $8)$, but not others $(36,37)$, and was not associated with CD in a large meta-analysis of many studies conducted by Zhang et al. (9). This polymorphism introduces a stop codon in CARD8 T48 that, because of its proximity to the T48 start site, would effectively result in loss of CARD8 expression. However, Bagnall et al. showed that individuals with the polymorphism express a functional CARD8 (T47) isoform, presumably by utilizing a start site downstream of the polymorphism that is not affected by the latter $(5,10)$. CARD8 T60, due to its alternative start site, has a P102I substitution that does not cause a stop codon as a result of this polymorphism. Nevertheless, we conducted studies of CARD8 T60 containing this substitution to investigate its possible effect on CARD8 T60 function and showed that it had no effect of CARD8 T60 interaction with NLRP3 or NLRP3 regulatory function. Therefore, even in individuals carrying a homozygous SNP at rs2043211, the expression of CARD8 T60 and CARD8 T47 plays a normal downregulatory role in the NLRP3 inflammasome.

Several recent publications have implicated NLRP3 phosphorylation in the regulation of NLRP3 inflammasome activation. Thus, Stutz et al. and Mortimer et al. reported that dephosphorylation of serine (S5 or S295) is necessary for NLRP3 activation, whereas Spalinger et al. reported that dephosphorylation of a tyrosine (Y861) has this function $(13,38,39)$. It was therefore logical to assume that CARD8 regulates NLRP3 inflammasome by affecting NLRP3 dephosphorylation. We investigated this possibility by assessing the effect of unmutated and mutated CARD8 T60 on NLRP3 serine phosphorylation and showed that, whereas unmutated CARD8 T60 was associated with NLRP3 having intact serine phosphorylation, mutated CARD8 T60 was associated with NLRP3 having diminished serine phosphorylation. Thus, loss of 
binding of CARD8 due to mutation was associated with loss of serine phosphorylation, both conditions in which the NLRP3 inflammasome is activated. These data suggest that CARD8 in an unmutated state retards serine dephosphorylation and thereby inhibits NLRP3 activation; however, additional studies will be necessary to demonstrate an actual cause and effect relation.

Data from several recent publications suggest that the NLRP3 inflammasome is regulated by polyubiquitination of NLRP3. Thus, Yan et al. (40) reported that K48-linked ubiquitination negatively regulated NLRP3 inflammasome activity by promoting the degradation of NLRP3. This idea was supported by Han et al. (41) and Song et al. (15), although they identified different ubiquitinases as operative compared with those identified by Yan et al. In addition, Py et al. (14) showed that deubiquitination of both K63and K48-linked polyubiquitin chains was required for NLRP3 inflammasome activation. A somewhat different view of the role of polyubiquitination in NLRP3 activation was suggested by Wei et al. (42), who showed that the Yersinia-type III secretion effector YopM promoted K63-linked polyubiquitination and activation of NLRP3; thus, in this case, K63-linked polyubiquitination was shown to be a positive regulator of the NLRP3 inflammasome. Our data showed that interaction of intact CARD8 with NLRP3 results in reduced K63- and K48-linked polyubiquitination of NLRP3; these data thus support the idea that intact CARD8 acts as a negative regulator of NLRP3, at least in part by its ability to promote NLRP3 K63- and K48-linked polyubiquitination. In contrast, mutant CARD8 does not maintain NLRP3 polyubiquitination and causes increased NLRP3 activation. It should be noted that serine phosphorylation and polyubiquitination of NLRP3 may be linked phenomena that operate in tandem to prevent NLRP3 activation.

In summary, these data provide what we believe is the first evidence that enhanced production of IL-1 $\beta$ due to an intrinsic abnormality of the NLRP3 inflammasome activity can be a primary cause of CD. This is contrary to the notion that IL-1 $\beta$ cannot in itself cause this form of GI inflammation and participates in the inflammatory process only in concert with other proinflammatory cytokines or the absence of regulatory cytokines. With the discovery that enhanced NLRP3 inflammasome activity can be a cause of CD and that patients with increased NLRP3 activity require therapy (such as anti-IL-1 $\beta$ administration) not ordinarily used in CD treatment, it is incumbent on gastroenterologists to consider the possibility that patients resistant to ordinary treatment may have a form of disease due to IL- $1 \beta$ that can therefore be treated with anti-IL-1 $\beta$-based therapies.

\section{Methods}

Human subjects. Peripheral blood from the proband patient with CD and his mother, father, and maternal aunt was drawn for whole-exome sequencing and other laboratory examinations. Blood was obtained from sex-matched healthy controls. Peripheral blood from another 4 patients with active $\mathrm{CD}$ was drawn for serum cytokine assay.

Reagents and constructs. Ultrapure LPS (L3012), ATP (tlrl-atpl), nigericin (tlrl-nig), poly(dA:dT) (tlrl-patn), flagellin (tlrl-epstfla-5), PMA (P8139), and MSU (tlrl-msu) were purchased from InvivoGen. TcdB toxin (6246-GT-020) was purchased from R\&D Systems. Disuccinimidyl suberate (DSS) crosslinker (S1885) was purchased from Sigma-Aldrich. Myc-DDK-tagged pCMV6 human CARD8 T60 construct, transcript variant 1, was purchased from Origene (RC230245). CARD8 V44I point mutation was introduced by using the Q5 Site-Directed Mutagenesis Kit (E0554S, New England Biolabs). The primers used were as follows: forward: 5'-ATTGACAATAGCATACGGGAA-3'; reverse: 5'-CAACAGTTTCCGTGATCCTTG-3'. Myc or Flag tag was deleted from pCMV6 constructs using the Q5 Site-Directed Mutagenesis Kit. The primers used were as follows: Myc deletion, forward: $5^{\prime}$-ATCCTGGATTACAAGGATGACGACGATAAGG-3', reverse: 5'-CTCGAGCGGCCGCGTACG-3'; Flag deletion, forward: 5'-GTTTAAACGGCCGGCCGC-3', reverse: 5'-TGCCAGATCCTCTTCTGAGATGAG-3'.

pcDNA3.1-CARD8 T48 was provided by Tetsuo Kubota (Tokyo Medical and Dental University, Tokyo, Japan) (5). CARD8 T48 was amplified by PCR and introduced into pCMV6 vector using the Quick-Fusion Cloning Kit (Biomake) with the following primers: forward: 5'-AGATCTGCCGCCGCGATCGCCATGATGAGACAGAGGCAGAGC-3'; reverse: 5'-CTCGAGCGGCCGCGTACGCGTCAAATTCTGCTGTCTAAGATA-3'.

Human cell isolation. PBMCs from both patients and healthy individuals were freshly isolated using LSM Lymphocyte Separation Medium (50494, MP Biomedicals). These cells were further isolated using the Human Pan Monocyte Isolation Kit (130-096-537, Miltenyi Biotec). The monocytes obtained were maintained in culture overnight before use.

Whole exome sequencing. We isolated DNA from whole blood using the salting-out method (QIAGEN), followed by phenol-chloroform extraction. We prepared whole genome libraries according to the KAPA HTP Library Construction Kit Protocol (Kapa Biosystems), performed exome capture according to Nimblegen's SeqCap EZ Library + UTR protocol, and sequenced the exomes thus obtained with Illumina HiSeq 2500. We mapped reads for all samples to human GRCh37 reference with BWA (43) v0.7.13 using the default settings and marked duplicate reads with Picard (http://broadinstitute.github.io/picard). Next, we applied Genome Analysis Toolkit (GATK) (44) v3.5 base quality score recalibration and indel realignment and performed SNP and INDEL discovery and genotyping across all samples simultaneously according to GATK Best Practices recommendations $(45,46)$. We normalized and decomposed variants with vt (47) v0.577, and annotated variants with variant effect predictor (48) v84. We filtered variants using GEMINI (49) v0.19.1 to obtain rare or novel variants with allele frequency of less than 0.1\% according to 1000 Genomes (http://www.internationalgenome. org/), the Exome Sequencing Project (http://evs.gs.washington.edu/ EVS/), and ExAC (http://exac.broadinstitute.org/). While applying different genetic model filters with GEMINI, we also filtered variants to remove any where minimum heterozygous allelic balance was less than 0.25 or that had a "High" Gene Damage Index for general Mendelian diseases (http://lab.rockefeller.edu/casanova/GDI; ref. 50).

Cell culture. HEK293 cells were purchased from ATCC (CRL-1573) and were maintained in IMDM medium containing 10\% FBS, $100 \mathrm{IU} /$ $\mathrm{ml}$ penicillin, and $1 \mathrm{mg} / \mathrm{ml}$ streptomycin. Human primary monocytes were maintained in RPMI 1640 medium containing 10\% FBS, 100 $\mathrm{IU} / \mathrm{ml}$ penicillin, $1 \mathrm{mg} / \mathrm{ml}$ streptomycin, and $50 \mu \mathrm{M} \beta$-mercaptoethanol. Human mDCs were allowed to differentiate in this RPMI 1640 medium mixture with recombinant granulocyte-macrophage CSF (GM-CSF) (20 ng/ml; Peprotech) and IL-4 (20 ng/ml; Peprotech) for 7 days. The cells were cultured in a humidified incubator with $5 \% \mathrm{CO}_{2}$ at $37^{\circ} \mathrm{C}$. The purity of $\mathrm{CD}_{11 \mathrm{C}^{+}}$cells was higher than $80 \%$ when tested by flow cytometry (data not shown). These cells were used for inflammasome activation experiments. 
Inflammasome activation. Human mDCs were primed for 6 hours with ultrapure LPS (100 ng/ml, Invivogen) prior to inflammasome activation. The cells were then treated with ATP ( $5 \mathrm{mM}, 30$ minutes), nigericin ( $1 \mu \mathrm{M}, 30$ minutes), or MSU ( $400 \mu \mathrm{g} / \mathrm{ml}, 2$ hours) to activate NLRP3 inflammasome. NLRC4 and AIM2 inflammasome were activated by transfection of flagellin ( $1 \mu \mathrm{g} / \mathrm{ml}, 2$ hours) or poly(dA:dT) (1 $\mu \mathrm{g} / \mathrm{ml}, 2$ hours) using Lipofectamine LTX (15338100, Thermo Fisher Scientific), respectively. TcdB toxin $(1 \mu \mathrm{g} / \mathrm{ml}, 2$ hours) was added to culture medium to activate pyrin inflammasome.

Real-time PCR. Total RNA was extracted from the human primary mDCs by using the RNeasy Mini Kit (QIAGEN, 74104). Reverse transcription of mRNA and synthesis of cDNA were performed using TaqMan reverse transcription reagents (Applied Biosystems). Realtime PCR was performed using SYBR Green qPCR Master Mix (Toyobo) and the 7900HT Fast Real-Time PCR system (Applied Biosyste$\mathrm{ms}$ ). Relative quantification of genes was achieved via normalization against $\beta$-actin. The primers used were as follows: GAPDH, 5'-ACGGATTTGGTCGTATTGGG-3' (forward), 5'-CATGGGTGGAATCATATTGGAAC-3' (reverse); pro-IL-1 13 , 5'-CACGATGCACCTGTACGATCA-3' (forward), 5'-GTTGCTCCATATCCTGTCCCT-3' (reverse); NLRP3, 5'-AAGGGCCATGGACTATTTCC-3' (forward), 5'-GACTCCACCCGATGACAGTT-3' (reverse); CARD8 T60, 5'-AACTGTGTTTCCTACCGAAACCC-3' (forward), 5'-AGGACATCGCTCTCTCAGGC-3' (reverse); ASC, 5'-AACCCAAGCAAGATGCGGAAG-3' (forward), 5'-TTAGGGCCTGGAGGAGCAAG-3' (reverse); aspase-1, 5'-AAGACCCGAGCTTTGATTGA-3' (forward), 5'-CCCTGCCAGGTAACTGTCTT-3' (reverse); and pyrin, 5'-ATCCAGAACATTCGGTCACC-3' (forward), 5'-CTTCCTTTCATGGGAGTCCTG-3' (reverse).

Western blots and immunoprecipitation. HEK293 cells or human primary $\mathrm{mDCs}$ were incubated in lysis buffer containing $50 \mathrm{mM}$ Tris $(\mathrm{pH}$ 7.5), $1 \% \mathrm{NP}-40,150 \mathrm{mM} \mathrm{NaCl}$, and protease inhibitor cocktail. After centrifugation at $13,000 \mathrm{~g}$ for 15 minutes at $4^{\circ} \mathrm{C}$, the supernatants were mixed with LDS loading buffer, heated at $100^{\circ} \mathrm{C}$ for 10 minutes, and then separated via LDS electrophoresis. The proteins were transferred onto nitrocellulose membranes and blocked with $5 \%$ fat-free milk in $1 \times$ Tris-buffered saline containing $0.05 \%$ Tween 20 and then probed with the corresponding primary antibodies to detect mature IL-1 $\beta$ and pro-IL-1 $\beta$ (sc-7884; clone H-153; Santa Cruz Biotechnology Inc.), NLRP3 (ALX804-881; clone cryo2; Enzo Life Sciences), ASC (sc-22514-R; clone N-15; Santa Cruz Biotechnology Inc.), mature and pro-caspase-1 (sc-515; clone C-20; Santa Cruz Biotechnology Inc.), CARD8 (sc-81213, clone 2108C2a; Santa Cruz Biotechnology Inc.; NB100-56181, Novus Biologicals), Myc tag (sc-40, clone 9E10; Santa Cruz Biotechnology Inc.), Flag tag (F1804, clone M2; Sigma-Aldrich), and phosphorylated NLRP3 (clone 9621S, Cell Signaling Technology). For mature caspase- 1 and IL-1 $\beta$ assays, the cell culture supernatants were harvested and concentrated via the protein precipitation approach, with the harvested protein pellets mixed with SDS loading buffer for the detection of caspase-1 p10 and IL-1 $1 \beta$ p17. After incubation with appropriate horseradish peroxidase-conjugated secondary antibodies, the immunoreactive bands were visualized using enhanced chemiluminescence reagent (K-12043-D10, Advansta).

ASC, NLRP3, or CARD8 oligomerization detection. The procedure for detecting ASC oligomerization was described previously (51). Briefly, human primary mDCs were primed with ultrapure LPS $(100 \mathrm{ng} / \mathrm{ml})$ for 6 hours. The cells were then activated with ATP $(5 \mathrm{mM})$ for 30 minutes. The culture supernatants were removed, and the cells were lysed with $0.2 \mathrm{ml}$ of lysis buffer containing $50 \mathrm{mM}$ Tris ( $\mathrm{pH} 7.5), 150 \mathrm{mM} \mathrm{NaCl}$,
$1 \% \mathrm{NP}-40$, and a protease inhibitor mixture. The cell lysates were put through a 21-gauge needle 10 times and then centrifuged at $12,000 \mathrm{~g}$ for 10 minutes at $4^{\circ} \mathrm{C}$. The resultant insoluble cell debris was washed 3 times with PBS and resuspended in $500 \mu \mathrm{l}$ PBS. DSS crosslinker $(0.8 \mathrm{mM}$, final concentration) was added to PBS containing the cell debris. The mixture was kept at $37^{\circ} \mathrm{C}$ for 30 minutes. The crosslinked cell debris was pelleted and solubilized in LDS loading buffer for Western blot detection of ASC oligomerization. To examine NLRP3 oligomerization, the mDCs were stimulated and lysed using the above methods. The cell lysates were mixed with DSS crosslinker (0.8 $\mathrm{mM}$, final concentration) and were incubated at $37^{\circ} \mathrm{C}$ for 30 minutes. Then the crosslinked cell lysates were mixed with LDS loading buffer for Western blot. For detecting CARD8 oligomerization, CARD8 T60 or T48 was transfected into HEK293 cells. The cells were harvested 48 hours later and lysed and crosslinked using the method for NLRP3 crosslinking.

ELISA. Serum samples were collected from CD patients and healthy controls. IL-1 $\beta$, IL- 6 , and TNF- $\alpha$ concentration was examined by ELISA (BD Biosciences). Supernatants of human PBMCs, primary monocytes, mDCs, or HEK293 cells were harvested, and IL-1 $\beta$ as well as IL-6 secretion was analyzed by ELISA. All procedures were performed according to the manufacturers' instructions.

Statistics. Data are presented as mean \pm SEM except where indicated otherwise. Statistical differences between 2 groups were analyzed using 2-tailed Students' $t$ test. The experiments with more than 2 groups of samples were analyzed using 1-way ANOVA followed by Dunnett's or Tukey's post hoc multiple comparisons. $P \leq 0.05$ was considered statistically significant.

Study approval. Written informed consent was obtained from all the patients and control individuals involved in this study. All procedures in this study were approved by the ethics committees of the University Hospitals Cleveland Medical Center, the Children's Hospital of Philadelphia, Stanford Medical Center (Stanford, California, USA), and the NIH. All human cell isolation was approved by the NIH Review Board for Human Subject Research.

\section{Author contributions}

LM performed studies, analyzed data, and wrote the manuscript. AK planned and analyzed studies. MS and AO provided genetic analysis. LA, JK, AA, and MY provided clinical care of patients. $\mathrm{KM}$ collected specimens and provided clinical care. IF provided clinical care of patients and planned and analyzed studies. MQ analyzed pathologic specimens. WS planned experiments, analyzed data, and wrote the manuscript.

\section{Acknowledgments}

This study was supported by Intramural NIAID Institute Project A1000345-35 and federal funds from the NCI, NIH, under contract no. HHSN261200800001E. This research was conducted under NIAID clinical project number 82-I-0183. The content of this publication does not necessarily reflect the views or policies of the Department of Health and Human Services, nor does mention of trade names, commercial products, or organizations imply endorsement by the US Government.

Address correspondence to: Warren Strober, NIH, 9000 Rockville Pike, Bldg. 10, CRC, 5 West Room 5-3940, Bethesda, Maryland 20892, USA. Phone: 301.496.6810; Email: wstrober@niaid.nih.gov. 
1. Jostins L, et al. Host-microbe interactions have shaped the genetic architecture of inflammatory bowel disease. Nature. 2012;491(7422):119-124.

2. Lewis GJ, et al. Genetic association between NLRP3 variants and Crohn's disease does not replicate in a large UK panel. Inflamm Bowel Dis. 2011;17(6):1387-1391.

3. Villani AC, et al. Common variants in the NLRP3 region contribute to Crohn's disease susceptibility. Nat Genet. 2009;41(1):71-76.

4. Cookson BT, Brennan MA. Pro-inflammatory programmed cell death. Trends Microbiol. 2001;9(3):113-114.

5. Ito S, Hara Y, Kubota T. CARD8 is a negative regulator for NLRP3 inflammasome, but mutant NLRP3 in cryopyrin-associated periodic syndromes escapes the restriction. Arthritis Res Ther. 2014;16(1):R52.

6. Cheung MS, Theodoropoulou K, Lugrin J, Martinon F, Busso N, Hofer M. Periodic fever with aphthous stomatitis, pharyngitis, and cervical adenitis syndrome is associated with a CARD8 variant unable to bind the NLRP3 inflammasome. J Immunol. 2017;198(5):2063-2069.

7. McGovern DP, et al. TUCAN (CARD8) genetic variants and inflammatory bowel disease. Gastroenterology. 2006;131(4):1190-1196.

8. Schoultz I, et al. Combined polymorphisms in genes encoding the inflammasome components NALP3 and CARD8 confer susceptibility to Crohn's disease in Swedish men. Am J Gastroenterol. 2009;104(5):1180-1188.

9. Zhang ZT, Ma XJ, Zong Y, Du XM, Hu JH, Lu GC. Is the CARD8 rs2043211 polymorphism associated with susceptibility to Crohn's disease? A meta-analysis. Autoimmunity. 2015;48(8):524-531.

10. Bagnall RD, Roberts RG, Mirza MM, Torigoe T, Prescott NJ, Mathew CG. Novel isoforms of the CARD8 (TUCAN) gene evade a nonsense mutation. Eur J Hum Genet. 2008;16(5):619-625.

11. Itan $Y$, et al. The mutation significance cutoff: gene-level thresholds for variant predictions. Nat Methods. 2016;13(2):109-110.

12. Bouchier-Hayes L, et al. CARDINAL, a novel caspase recruitment domain protein, is an inhibitor of multiple NF-kB activation pathways. J Biol Chem. 2001;276(47):44069-44077.

13. Mortimer L, Moreau F, MacDonald JA, Chadee K. NLRP3 inflammasome inhibition is disrupted in a group of auto-inflammatory disease CAPS mutations. Nat Immunol. 2016;17(10):1176-1186.

14. Py BF, Kim MS, Vakifahmetoglu-Norberg H, Yuan J. Deubiquitination of NLRP3 by BRCC3 critically regulates inflammasome activity. $\mathrm{Mol} \mathrm{Cell}$. 2013;49(2):331-338.

15. Song $\mathrm{H}$, et al. The E3 ubiquitin ligase TRIM31 attenuates NLRP3 inflammasome activation by promoting proteasomal degradation of NLRP3. Nat Commun. 2016;7:13727.

16. Razmara M, et al. CARD-8 protein, a new CARD family member that regulates caspase- 1 activation and apoptosis. J Biol Chem. 2002;277(16):13952-13958.

17. Coccia $\mathrm{M}$, et al. IL-1 $\beta$ mediates chronic intestinal inflammation by promoting the accumulation of IL-17A secreting innate lymphoid cells and CD4(+)
Th17 cells. J Exp Med. 2012;209(9):1595-1609.

18. Ligumsky M, Simon PL, Karmeli F, Rachmilewitz D. Role of interleukin 1 in inflammatory bowel disease--enhanced production during active disease. Gut.1990;31(6):686-689.

19. McAlindon ME, Hawkey CJ, Mahida YR. Expression of interleukin 1 beta and interleukin 1 beta converting enzyme by intestinal macrophages in health and inflammatory bowel disease. Gut. 1998;42(2):214-219.

20. Bauer C, Duewell P, Lehr HA, Endres S, Schnurr M. Protective and aggravating effects of Nlrp3 inflammasome activation in IBD models: influence of genetic and environmental factors. Dig Dis. 2012;30(suppl 1):82-90.

21. Dupaul-Chicoine J, et al. Control of intestinal homeostasis, colitis, and colitis-associated colorectal cancer by the inflammatory caspases. Immunity. 2010;32(3):367-378.

22. Lebeis SL, Powell KR, Merlin D, Sherman MA, Kalman D. Interleukin-1 receptor signaling protects mice from lethal intestinal damage caused by the attaching and effacing pathogen Citrobacter rodentium. Infect Immun. 2009;77(2):604-614.

23. Zaki MH, Boyd KL, Vogel P, Kastan MB, Lamkanfi M, Kanneganti TD. The NLRP3 inflammasome protects against loss of epithelial integrity and mortality during experimental colitis. Immunity. 2010;32(3):379-391.

24. Hirota SA, et al. NLRP3 inflammasome plays a key role in the regulation of intestinal homeostasis. Inflamm Bowel Dis. 2011;17(6):1359-1372.

25. Neudecker V, et al. Myeloid-derived miR-223 regulates intestinal inflammation via repression of the NLRP3 inflammasome. J Exp Med. 2017;214(6):1737-1752.

26. Yao X, et al. Remodelling of the gut microbiota by hyperactive NLRP3 induces regulatory $\mathrm{T}$ cells to maintain homeostasis. Nat Commun 2017;8(1):1896.

27. Yao Y, et al. Tr1 cells, but not Foxp $3^{+}$regulatory T cells, suppress NLRP3 inflammasome activation via an IL-10-dependent mechanism. J Immunol. 2015;195(2):488-497.

28. Zhang J, Fu S, Sun S, Li Z, Guo B. Inflammasome activation has an important role in the development of spontaneous colitis. Mucosal Immunol. 2014;7(5):1139-1150.

29. Liu L, et al. The pathogenic role of NLRP3 inflam masome activation in inflammatory bowel diseases of both mice and humans. JCrohns Colitis. 2017;11(6):737-750.

30. Carvalho FA, et al. Interleukin-1 $\beta$ (IL-1 $\beta$ ) promotes susceptibility of Toll-like receptor 5 (TLR5) deficient mice to colitis. Gut. 2012;61(3):373-384.

31. Shouval DS, et al. Interleukin $1 \beta$ mediates intestinal inflammation in mice and patients with interleukin 10 receptor deficiency. Gastroenterology. 2016;151(6):1100-1104.

32. Opipari A, Franchi L. Role of inflammasomes in intestinal inflammation and Crohn's disease. Inflamm Bowel Dis. 2015;21(1):173-181.

33. Galeotti C, et al. Efficacy of interleukin-1-targeting drugs in mevalonate kinase deficiency. Rheumatology (Oxford). 2012;51(10):1855-1859.
34. de Luca A, et al. IL-1 receptor blockade restores autophagy and reduces inflammation in chronic granulomatous disease in mice and in humans. Proc Natl Acad Sci U S A. 2014;111(9):3526-3531.

35. Canna SW, et al. Life-threatening NLRC4-associated hyperinflammation successfully treated with IL-18 inhibition. J Allergy Clin Immunol. 2017;139(5):1698-1701.

36. Fisher SA, et al. Combined evidence from three large British Association studies rejects TUCAN/ CARD8 as an IBD susceptibility gene. Gastroenterology. 2007;132(5):2078-2080.

37. Büning $\mathrm{C}$, et al. No association of the CARD8 (TUCAN) c.30T $>$ A (p.C10X) variant with Crohn's disease: a study in 3 independent European cohorts. Inflamm Bowel Dis. 2008;14(3):332-337.

38. Stutz A, et al. NLRP3 inflammasome assembly is regulated by phosphorylation of the pyrin domain. JExp Med. 2017;214(6):1725-1736.

39. Spalinger MR, et al. NLRP3 tyrosine phosphorylation is controlled by protein tyrosine phosphatase PTPN22. JClin Invest. 2016;126(5):1783-1800.

40. Yan Y, et al. Dopamine controls systemic inflammation through inhibition of NLRP3 inflammasome. Cell. 2015;160(1-2):62-73.

41. Han S, et al. Lipopolysaccharide primes the NALP3 inflammasome by inhibiting its ubiquitination and degradation mediated by the SCFFBXL2 E3 ligase. J Biol Chem. 2015;290(29):18124-18133.

42. Wei C, et al. The Yersinia Type III secretion effector YopM is an E3 ubiquitin ligase that induced necrotic cell death by targeting NLRP3. Cell Death Dis. 2016;7(12):e2519.

43. Li H, Durbin R. Fast and accurate short read alignment with Burrows-Wheeler transform. Bioinformatics. 2009;25(14):1754-1760.

44. McKenna A, et al. The Genome Analysis Toolkit: a MapReduce framework for analyzing nextgeneration DNA sequencing data. Genome Res. 2010;20(9):1297-1303.

45. DePristo MA, et al. A framework for variation discovery and genotyping using nextgeneration DNA sequencing data. Nat Genet. 2011;43(5):491-498.

46. Van der Auwera GA, et al. From FastQ data to high confidence variant calls: the Genome Analysis Toolkit best practices pipeline. Curr Protoc Bioinformatics. 2013;43:11.10.1-11.1033.

47. Tan A, Abecasis GR, Kang HM. Unified representation of genetic variants. Bioinformatics. 2015;31(13):2202-2204.

48. McLaren $W$, et al. The ensembl variant effect predictor. Genome Biol. 2016;17(1):122.

49. Paila U, Chapman BA, Kirchner R, Quinlan AR. GEMINI: integrative exploration of genetic variation and genome annotations. PLoS Comput Biol. 2013;9(7):e1003153.

50. Itan Y, et al. The human gene damage index as a gene-level approach to prioritizing exome variants. Proc Natl Acad Sci U S A. 2015;112(44):13615-13620.

51. Mao L, et al. Pathogenic fungus Microsporum canis activates the NLRP3 inflammasome. Infect Immun. 2014;82(2):882-892. 